\title{
Deep optical imaging of radio selected BL Lacertae objects ${ }^{\star}$
}

\author{
T. Pursimo ${ }^{1}$, K. Nilsson ${ }^{1}$, L. O. Takalo ${ }^{1}$, A. Sillanpää ${ }^{1}, J$. Heidt ${ }^{2}$, and H. Pietilä ${ }^{1}$ \\ 1 Tuorla Observatory, University of Turku, 21500 Piikkiö, Finland \\ 2 Landessternwarte, Königstuhl, 69117 Heidelberg, Germany
}

Received 29 March 2000 / Accepted 19 October 2001

\begin{abstract}
Deep imaging data on radio-selected BL Lacertae objects are presented. The complete 1 Jy sample consists of 37 objects. Altogether 25 objects were observed which span a large range of redshifts (up to $z=1.34$ ) with 12 objects having $z>0.5$. In addition, three objects are located at an unknown distance. Altogether in eleven objects the host galaxy could be resolved. All nine objects with $z \lesssim 0.4$ were resolved and all eleven objects with $z \gtrsim 0.7$ or unknown redshift were unresolved. In all (six) low- $z(z \lesssim 0.2)$ objects, an elliptical host galaxy was preferred. Of the remaining objects, only in one case $(2007+777)$ did a disc-type galaxy give better fit than elliptical, but in this case neither can be preferred. The absolute brightness of the host galaxies (median $\left.M_{R}=-23.82\right)$ is similar to the findings of X-ray selected BL Lacs and low redshift radio galaxies. Also the sizes (median $r_{\mathrm{e}}=14.8 \mathrm{kpc}$ ) of the galaxies are similar. This supports the idea that (at least low redshift) BL Lacs belong to one family of objects. These results agree well with other recent studies on BL Lacs. There is a strong correlation between the brightness of the active nucleus and redshift. At low redshift radio and X-ray selected BL Lacs have similar brightnesses. No (or only weak) correlation was found between the brightness of the nucleus and the host galaxy.
\end{abstract}

Key words. BL Lacertae objects: general - galaxies: nuclei - galaxies: structure

\section{Introduction}

BL Lac objects are a subclass of active galactic nuclei (AGN) in which broad bands emission is dominated by synchrotron radiation from radio to X-ray bands and the higher energy component by (possibly) inverse-Compton scattering. BL Lacs are characterised by no or only weak emission lines, rapid variability from the radio regime up to $\mathrm{TeV}$ energies, high and variable polarization $\left(P_{\mathrm{opt}}>\right.$ $3 \%$ ) and apparent superluminal motion in the VLBI structures. The rapid variability is thought to be due to relativistic beaming (e.g. Blandford \& Rees 1978).

Since the 1980's BL Lacs have been divided roughly into two separate families, based on the discovery frequency: radio and X-ray selected BL Lacs (RBLs and XBLs, respectively). A more physical division, based on the peak frequency of the synchrotron component, was proposed by Padovani \& Giommi (1995). According to this

Send offprint requests to: T. Pursimo,

e-mail: tpursimo@utu.fi

* Based on observations made with the Nordic Optical Telescope, operated on the island of La Palma jointly by Denmark, Finland, Iceland, Norway and Sweden, in the Spanish Observatorio del Roque de los Muchachos of the Instituto de Astrophysica de Canarias. model most RBLs are classified as low-frequency peaked BL Lacs (LBLs) and most XBLs as high-frequency peaked BL Lacs (HBLs). Indeed, many properties show discontinuity from HBLs to LBLs (e.g. polarization properties, broad band spectral indices, the peak frequency of the spectral energy distribution [SED] in $\nu S_{\nu}$ ). However, recent sensitive sky surveys (radio, X-ray) have enabled one to construct new BL Lac samples, showing properties intermediate between LBLs and HBLs (e.g. LaurentMuehleisen et al. 1999; Pursimo et al. 2001). This supports the idea that there is a single population of BL Lacs, with the extreme ends being HBLs and LBLs (for this unification of BL Lacs see Padovani 1999). Furthermore, based on the unifying scheme of radio loud AGNs, it is generally thought that the parent population of BL Lacs are FR I radio galaxies (see the review by Urry \& Padovani 1995). Gravitational microlensing proposed by Ostriker \& Vietri (1985) has not proven to be the general explanation of the BL Lac-phenomenon (Padovani 1999), but it might be present in some cases (Stocke \& Rector 1997). This might cause unknown biases to the bulk properties of the $1 \mathrm{Jy}$ sample.

To test the unification schemes, isotropic properties (e.g. host galaxies, close environments and clustering) of different classes must be compared. Deep imaging can be 
used to study the properties of the host galaxies in order to compare: 1) different subclasses of BL Lacs and 2) BL Lacs, quasars and radio galaxies. To minimize selection effects, the tests require large and complete samples. These studies can also illuminate the triggering mechanism (e.g. tidal perturbations) of the active phase of a galaxy.

Previous imaging studies of BL Lacs have shown that the host galaxies have, almost exclusively, de Vaucouleurstype surface brightness profiles $\left(\mu(r) \propto r^{-1 / 4}\right)$, as elliptical galaxies do. The host galaxy is typically about $1 \mathrm{mag}-$ nitude brighter than $M_{R}^{*}$-galaxy $\left(M_{R}^{*}=-22.4\right.$; Efstathiou et al. 1988, converted from $M_{B_{\mathrm{T}}}^{*}$ assuming $B-R=1.56$ ) of the Schechter luminosity function (e.g. Heidt 1999 and references therein, Falomo \& Kotilainen 1999, hereafter FK99; Urry et al. 2000, hereafter U00) which is between the typical FRI and FRII host galaxy magnitudes. In some cases a disc-type profile has been preferred but later independent observations put most of these results into question (OQ530 Abraham et al. 1991, hereafter A91; Stickel et al. 1993, hereafter S93; MS0205.7+3509 Stocke et al. 1995; Falomo et al. 1997b).

The complete $1 \mathrm{Jy}$ sample of BL Lacs has its roots in the $5 \mathrm{GHz}$ catalogue (Kühr et al. 1981). The selection criteria were flat radio spectrum, optically brighter than $m_{B} \sim 20$ on the Palomar plates (POSS), and the strongest rest frame optical emission line width less than $5 \AA$ (Stickel et al. 1991). The original complete 1 Jy sample consisted of 34 objects (Stickel et al. 1991). Later this was revised by Stickel et al. (1994) and it contains now 37 BL Lac objects.

The aim of this work is to study the properties of the 1 Jy BL Lac's host galaxies and active nuclei. Deep images enable one to determine the morphology of the nearby objects and at least resolve more distant ones. A further aim of the nearby objects is to obtain an accurate brightness of the nucleus, which is uncontaminated by the host galaxy. These results will be compared to a recent study of a sample of XBLs (northern EMSS and Slew Survey BL Lacs), which was studied by FK99 using deep, high resolution Nordic Optical Telescope (NOT) images with instrumentation and method of analysis similar to the present work.

The paper is organised as follows. In Sect. 2 the observations and data reductions are briefly described. The results are presented in Sect. 3 and the summary is given in Sect. 4. Comments on individual objects are given in Appendix A and the dependence of the deepness of the image and the galaxy properties are compared in Appendix B. Throughout the paper $H_{0}=$ $50 \mathrm{~km} \mathrm{~s}^{-1} \mathrm{Mpc}^{-1}$ and $q_{0}=0$ cosmology has been adopted.

\section{Observations, data reductions and analysis}

The observations were carried out at the $2.56 \mathrm{~m} \mathrm{NOT}^{1}$ telescope in two observing runs (Oct. 95 and July 96)

\footnotetext{
1 For more information on NOT see http://www.not.iac.es/
}

with some additional data from other observing runs at NOT. Observations were made using the BroCam1 CCD (Tektronix $1024^{2}, 0^{\prime \prime} 176$ pixel $^{-1}$ ) except in June 96 when the BroCam2 CCD (Loral, 2048 ${ }^{2}, 0^{\prime \prime} 11$ pixel $^{-1}$ ) was used. The log of observations and basic information on the objects is given in Table 1 . The typical integration time was $\sim 5000 \mathrm{~s}$ with individual exposure times from 30 to $1200 \mathrm{~s}$ in order to keep the core unsaturated. The seeing was generally very good $\left(0.0^{\prime \prime}-1{ }^{\prime \prime} 0\right)$, with the average $F W H M$ being $\sim 0^{\prime \prime} 77$. All observations were made through $R$-band filter and photometric calibration of the July 96 run was carried out using standard stars from Landolt (1983, 1993). The Oct. 95 run non-photometric data were calibrated using either calibrated comparison stars from the same field (Fiorucci \& Tosti 1996; Fiorucci et al. 1998; Smith et al. 1985; Villata et al. 1998) or short exposures from other observing runs at NOT.

Altogether $70 \%$ (25 out of 37 ) of the 1 Jy BL Lac objects were observed. Six of the unobserved objects ${ }^{2}$ have $\delta<-26^{\circ}$, thus they are not accessible to NOT. In the analysis (Sect. 3) the results of AO 0235+164 (Nilsson et al. 1996) were also used. Despite the incompleteness of the observed sample, we believe that the derived parameters are representative compared to the whole $1 \mathrm{Jy}$ sample. Only one $z<0.5$ remained unobserved. Individual case studies based on these observations of nearby environments (PKS 0139-097, Heidt et al. 1996; 3C371, Nilsson et al. 1997) and two dimensional analysis on Mkn 501 (Nilsson et al. 1999, hereafter N99) have already been published.

\subsection{Data analysis}

The CCD frames were reduced using standard IRAF routines (debiasing and flatfielding using twilight flats). In all images the background was constant within $1 \%$ or better. The one-dimensional analysis (azimuthally averaged radial profiles) was done using the PROFIT-package (see a more detailed description in Scarpa et al. 2000, hereafter SU00) in a similar way to FK99. The first step in the analysis was to estimate the background by averaging several boxes near the object (or field star) but far outside the extracted profile. After that, close companions were masked and the radial profiles (the object and field stars) were extracted using PROFIT. The modeling of the observed surface brightness profile was carried out using simultaneous scaling of the point spread function (PSF) and the seeing (PSF) convolved galaxy model (either de Vaucouleurs $\mu(r) \propto r^{-1 / 4}$ or exponential disc $\left.\mu(r) \propto r^{-1}\right)$.

For a proper fitting, an accurate PSF is needed. Ideally, the PSF is constructed using the average of several isolated field stars situated in the object's frame and having similar brightness to the object. However, typically only one or two such stars were found.

\footnotetext{
${ }^{2}$ Missing northern objects are: $0218+357, z=0.685$; $0828+493, z=0.548 ; 0954+658, z=0.367 ; 2029+121, z=$ $1.215 ; 2150+173, z=$ unknown).
} 
Table 1. Log of observations. The columns are; name of the object, redshift, $K$-correction for the host galaxy, galactic extinction, integration time, seeing, date of observation, sky brightness and comments. The redshifts are taken from Stickel et al. (1993), except 0138-097 and 0454+844 from Stocke \& Rector (1997); 0814+425, Lawrence et al. (1996) and 2131-021, Drinkwater et al. (1997).

\begin{tabular}{lcccrrrll}
\hline Name & $z$ & $K$-corr. & $A_{R}$ & $\begin{array}{r}t_{\text {int }} \\
\text { s. }\end{array}$ & $\begin{array}{r}F W H M \\
{\left[^{\prime \prime}\right]}\end{array}$ & $\begin{array}{r}\text { Date } \\
\text { ddmmyy }\end{array}$ & $\begin{array}{l}\text { sky } \\
{\left[\mathrm{mag} /{ }^{\prime 2}{ }^{2}\right]}\end{array}$ & Comments \\
\hline $0048-097$ & $\ldots$ & $\ldots$ & 0.17 & 960 & 0.70 & 23.10 .95 & 20.7 & calibration uncertain \\
$0138-098$ & 0.733 & 1.46 & 0.14 & 6300 & 0.77 & 22.10 .95 & 20.41 & PSF uncertain \\
$0454+844$ & 1.340 & 3.08 & 0.29 & 5700 & 0.81 & 20.10 .95 & 20.64 & $z_{\text {abs }}$ \\
$0716+714$ & $\ldots$ & $\ldots$ & 0.17 & 2120 & 1.02 & 23.10 .95 & 20.75 & PSF uncertain \\
$0735+178$ & 0.424 & 0.54 & 0.22 & 6760 & 0.65 & 21.10 .95 & 20.49 & z abs \\
$0814+425$ & 1.252 & 2.85 & 0.22 & 5100 & 0.77 & 21.10 .95 & 20.23 & redshift uncertain \\
$0820+225$ & 0.951 & 2.07 & 0.20 & 4800 & 1.00 & 23.10 .95 & 20.23 & 4C22.21 \\
$0823+033$ & 0.506 & 0.73 & 0.17 & 3300 & 0.73 & 4.1 .98 & 21.06 & OJ038 \\
$0851+202$ & 0.306 & 0.33 & 0.14 & 5640 & 0.67 & 4.1 .98 & 20.76 & OJ287 \\
$1147+245$ & $\ldots$ & $\ldots$ & 0.10 & 3600 & 0.85 & 19.6 .96 & 20.68 & OM280, PSF uncertain \\
$1308+326$ & 0.997 & 2.19 & 0.05 & 6000 & 0.86 & 8.7 .96 & 21.26 & \\
$1418+546$ & 0.152 & 0.15 & 0.05 & 6000 & 0.67 & 10.7 .96 & 20.82 & OQ530 \\
$1514-241$ & 0.049 & 0.04 & 0.41 & 3180 & 0.74 & 12.7 .96 & 20.51 & AP Lib \\
$1538+149$ & 0.605 & 1.05 & 0.14 & 4980 & 0.78 & 9.7 .96 & 21.18 & 4C14.60 \\
$1652+398$ & 0.033 & 0.03 & 0.08 & 3680 & 0.69 & 8.7 .96 & 21.49 & Mkn501 \\
$1749+096$ & 0.320 & 0.35 & 0.44 & 2160 & 0.77 & 11.7 .96 & 21.22 & 4C09.57, OT091 \\
$1749+701$ & 0.770 & 1.57 & 0.20 & 5460 & 0.61 & 21.10 .95 & 20.52 & \\
$1803+784$ & 0.684 & 1.30 & 0.20 & 5520 & 0.83 & 22.10 .95 & 20.32 & \\
$1807+698$ & 0.051 & 0.04 & 0.22 & 1530 & 0.67 & 12.7 .96 & 21.24 & 3C371 \\
$1823+568$ & 0.664 & 1.23 & 0.20 & 2400 & 0.86 & 11.7 .96 & 21.52 & 4C56.37 \\
$2007+777$ & 0.342 & 0.38 & 0.41 & 5100 & 0.86 & 9.7 .96 & 21.08 & \\
$2131-021$ & 1.285 & 2.94 & 0.20 & 5400 & 0.67 & 21.10 .95 & 20.47 & 4C-02.81 \\
$2200+420$ & 0.069 & 0.06 & 0.86 & 5400 & 0.79 & 20.10 .95 & 20.58 & BL Lacertae \\
$2240-260$ & 0.774 & 1.59 & 0.08 & 2460 & 0.95 & 8.7 .96 & 20.67 & \\
$2254+074$ & 0.194 & 0.20 & 0.24 & 1320 & 0.60 & 22.10 .95 & 20.35 & OY091 \\
\hline
\end{tabular}

In three cases the brightest unsaturated field star was more than $1.5 \mathrm{mag}$ fainter than the object, hence the PSF was constructed from other fields observed during the same run with similar seeing conditions (see Appendix A). The PSF appeared to be stable across the image. Resolved objects were modeled for both de Vaucouleurs and disc type profile (Table 2). The upper limit of the host galaxy's magnitude of the unresolved objects (Table 3 ) were determined using a fixed $1^{\prime \prime}$ effective radius, which corresponds 8 to $12 \mathrm{kpc}$ at the objects' redshifts. However all of these objects were classified as unresolved and moreover only one of these has a measured redshift. The main conclusions of the paper were drawn from the resolved objects, hence the conclusions remain the same.

As a check of the fitting procedure, previously analysed archived NTT data were re-analysed. Images of $2254+074$ (Falomo 1996) were retrieved and the same an analysis was performed as for the NOT data. The results using the PROFIT package are similar to those of Falomo, $r_{\mathrm{e}}=$ $3 . \prime 74, m_{\mathrm{AGN}}=17.36, m_{\mathrm{deV}}=16.70$ (see Table 4 below).

Galactic absorption $\left(A_{R}\right)$ was estimated using the formula by Schull \& van Steenberg (1985) after estimating the HI column density from Stark et al. (1992). The cosmological $K$-correction was derived from Coleman et al. (1980). It should be noted that these data and FK99 form a uniform sample regarding the instrumentation, data analysis, selected cosmology and $A_{R}$ and $K$-corrections. This enables a direct comparison between the current $1 \mathrm{Jy}$ and FK99 EMSS/Slew samples.

\section{Results and discussion}

Out of 25 objects, 11 were resolved, and are presented in Fig. 1 along with the observed radial profiles. The fitted model (PSF, galaxy model and the sum of these two) and the model parameters are shown in Table 2. The gray-scale images of the objects will be published in a subsequent paper on the study of nearby environments of the $1 \mathrm{Jy}$ sample (Pursimo et al. in preparation). In $6(z<0.2)$ cases the type of the host galaxy could be accurately defined. In the remaining 5 resolved objects the signal to noise ratio is not high enough to discriminate a de Vaucouleurs model from a disc model, yet in all but one case de Vaucouleurs' model was preferred. For this exception, 2007+777, a disctype host gives a smaller $\chi^{2}$-value than de Vaucouleurstype, however neither model gives a reasonable fit (see Fig. 1 and Appendix A). In one case (OJ287) the detection of the host galaxy was marginal. For the analysis below, the de Vaucouleurs model was always used.

For the nearby objects $(z \lesssim 0.2)$ the morphology of the host galaxy is well defined, yet fluctuations across the whole profile are seen. As an example Fig. 2 shows the residuals of the best fit model for $1807+698$. In the core 
Table 2. Observed R-band BL Lac host galaxy properties. The first line gives the best fit de Vaucouleurs model, followed by the best fit disc model. The magnitude of the point source, the magnitude of the model galaxy and the size of the galaxy with errors are given in columns two, four and six, respectively, followed by the surface brightness at $r_{\mathrm{e}}$. Column nine gives the $\chi^{2}$-statistics of the fit. For some objects also only point source best fit statistics are also given.

\begin{tabular}{|c|c|c|c|c|c|c|c|c|c|}
\hline Object & $m_{\mathrm{AGN}}$ & err & $\begin{array}{l}m_{\text {host }}^{\text {deV }} \\
m_{\text {host }}^{\text {disc }}\end{array}$ & err & $r_{\mathrm{e}}\left[^{[\prime}\right]$ & err & $\begin{array}{c}\mu \\
{\left[\mathrm{mag} /\left({ }^{\prime \prime 2}\right)\right]}\end{array}$ & $\begin{array}{c}\chi^{2} / \text { d.o.f. } \\
\chi^{2} / \text { d.o.f. }\end{array}$ & Comm. \\
\hline \multirow[t]{2}{*}{$0851+202$} & 15.32 & 0.01 & 18.91 & 0.17 & 0.99 & 0.29 & 22.18 & 8.76 & $\mathrm{AGN}=15.29, \chi^{2}=20.58$ \\
\hline & 15.30 & 0.01 & 19.63 & 0.12 & 2.45 & 0.28 & & 9.18 & \\
\hline \multirow[t]{2}{*}{$1418+546$} & 15.69 & 0.01 & 16.04 & 0.01 & 2.32 & 0.06 & 21.04 & 5.73 & \\
\hline & 15.40 & 0.01 & 16.73 & 0.01 & 3.39 & 0.02 & & 16.63 & \\
\hline \multirow[t]{2}{*}{$1514-241$} & 15.57 & 0.01 & 14.29 & 0.01 & 6.72 & 0.04 & 21.81 & 14.79 & \\
\hline & 14.84 & 0.01 & 14.84 & 0.01 & 5.71 & 0.01 & & 207.71 & \\
\hline \multirow[t]{2}{*}{$1538+149$} & 17.54 & 0.01 & 19.86 & 0.18 & 3.99 & 2.11 & 26.25 & 0.68 & $\mathrm{AGN}=17.49, \chi^{2}=59.37$ \\
\hline & 17.52 & 0.0 & 20.60 & 0.09 & 2.45 & 0.25 & & 0.94 & \\
\hline \multirow[t]{2}{*}{$1652+398$} & 14.08 & 0.01 & 12.42 & 0.01 & 17.21 & 0.19 & 22.00 & 6.66 & \\
\hline & 13.70 & 0.01 & 13.03 & 0.01 & 11.50 & 0.01 & & 780.75 & \\
\hline \multirow[t]{2}{*}{$1749+096$} & 19.47 & 0.07 & 18.70 & 0.07 & 1.35 & 0.12 & 22.69 & 0.93 & \\
\hline & 18.85 & 0.03 & 19.53 & 0.10 & 1.63 & 0.18 & & 0.78 & \\
\hline \multirow[t]{2}{*}{$1807+698$} & 14.70 & 0.01 & 14.17 & 0.01 & 10.76 & 0.14 & 22.71 & 10.72 & \\
\hline & 14.45 & 0.01 & 14.75 & 0.01 & 7.17 & 0.03 & & 163.13 & \\
\hline \multirow[t]{2}{*}{$1823+568$} & 18.10 & 0.01 & 19.75 & 0.10 & 2.61 & 0.56 & 25.21 & 1.79 & \\
\hline & 18.04 & 0.01 & 20.51 & 0.14 & 2.41 & 0.55 & & 1.97 & \\
\hline \multirow[t]{2}{*}{$2007+777$} & 17.89 & 0.01 & 18.89 & 0.05 & 2.89 & 0.29 & 24.58 & 26.61 & \\
\hline & 17.89 & 0.01 & 19.27 & 0.09 & 1.14 & 0.09 & & 6.09 & \\
\hline \multirow[t]{2}{*}{$2200+420$} & 14.92 & 0.02 & 15.03 & 0.01 & 6.63 & 0.20 & 22.57 & 2.75 & \\
\hline & 14.65 & 0.01 & 15.63 & 0.01 & 6.26 & 0.07 & & 25.53 & \\
\hline \multirow[t]{2}{*}{$2254+074$} & 16.39 & 0.01 & 16.52 & 0.01 & 4.57 & 0.24 & 23.21 & 1.64 & \\
\hline & 16.16 & 0.01 & 17.07 & 0.02 & 3.75 & 0.07 & & 9.94 & \\
\hline
\end{tabular}

$\left(r \lesssim 3^{\prime \prime}\right)$ the main contribution of the flux comes from the active nucleus (PSF) of the BL Lac object. At larger radii the PSF's surface brightness is well below the galaxy's value and the fitting depends only on $m_{\text {host }}$ and $r_{\mathrm{e}}$. The residuals could arise from the fitting process or perhaps the one dimensional profile does not follow the pure de Vaucouleurs law. In the latter case this could indicate either different $\beta$-values (e.g. Caon et al. 1993) or an extra component near the core of the galaxy. Recently nuclear discs have been detected in some FRI host galaxies (Jaffe et al. 1993; de Juan et al. 1996), but so far detection of inner discs are fairly rare in BL Lac objects (PKS 0548-322, Falomo et al. 1995; 1ES 1959+650, Heidt et al. 1999a and PKS 1413+135, McHardy et al. 1991). Note that, contrary to other objects, PKS $1413+135$ has a large disc and a small bulge. The extra flux in the tail could be a sign of the host galaxy's morphological peculiarity (e.g. tidal interaction, apparently the largest excess flux is in 1514-241 and 1807+698; both have nearby companions and show signs of interaction, S93).

The radial profiles of the unresolved objects are presented in Fig. 3. An object was classified as unresolved if the F-test suggest better or equally good fit for a pure point source than a PSF+de Vaucouleurs law or if the object does not follow the Kormendy relation (see below). The magnitudes of the active nucleus and upper limits of the host galaxies are shown in Table 3 . Virtually all objects with $z \lesssim 0.6$ were resolved but on the other hand all objects with $z \gtrsim 0.7$ were unresolved. This suggests that unresolved objects with unknown or tentative redshift are distant. For the objects with unknown redshift the minimum distance can be estimated using the lower limit of the host galaxy magnitude and the Hubble diagram (FK99 in their Fig. 9). However, the scatter in the diagram is quite large and e.g. in the case of $0851+202$ (OJ287) the expected redshift for a 19 mag host galaxy would be about 0.4 (vs. observed 0.306). The estimated redshifts are (conservative estimates): $z>0.2$ for $0048-097 ; z>0.15$ for $0716+714$ and $z>0.35$ for $1147+245$.

\subsection{Comparison to previous ground based studies}

Table 4 compares the results of previous ground-based studies and this work. In general the agreement is good, though there is some scatter in both $m_{\text {host }}$ and $r_{\mathrm{e}}$. In some cases the differences are significant, which are possibly due to the differences in the analysing methods (e.g. one or two-dimensional fitting or the methods of minimizing the $\chi^{2}$ ), quality and the deepness of the image. In the profile fitting the main difference between disc - and de Vaucouleurs - models comes from near the core or at the tail of the wings. The wings close to the $r_{\mathrm{e}}$ have similar slopes, hence high resolution and/or deep images are needed to discriminate the galaxy type. Finally, if there is an extra component in the core (as proposed in e.g. 

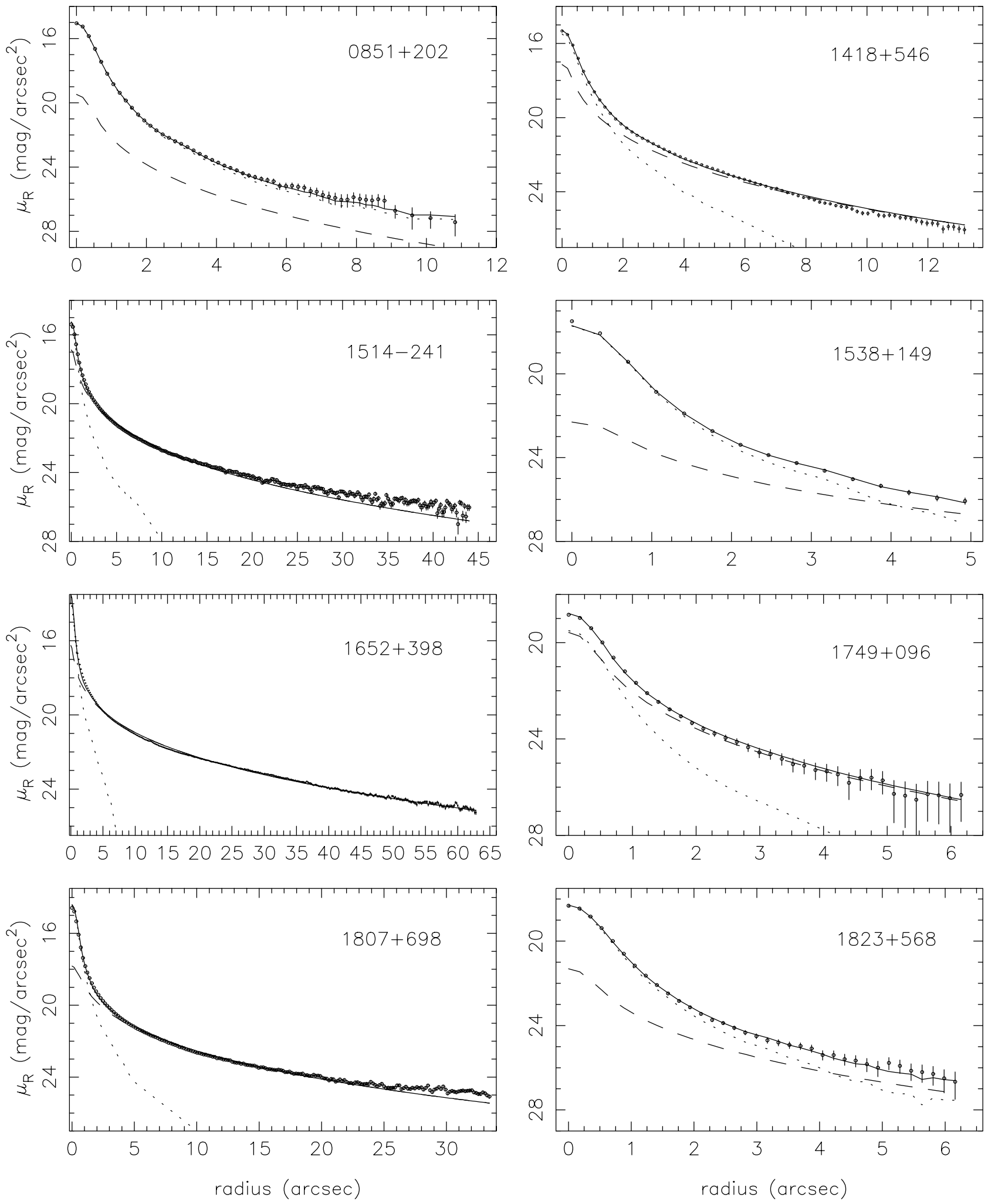

Fig. 1. The observed azimuthally averaged radial profiles of the resolved 1 Jy BL Lac objects. Filled dots represents the observed profile superimposed with the best fit of the total model (solid line), scaled PSF (short dashed line) and de Vaucouleurs galaxy model (long dashed line). Note that for $2007+777$ both best fit, elliptical and disc, models are presented.

PKS 0548-322, Falomo et al. 1995) the tail of the profile represents the main body of the galaxy profile while near the core the flux is a sum of the AGN-nucleus, host galaxy, and the "extra" component. 

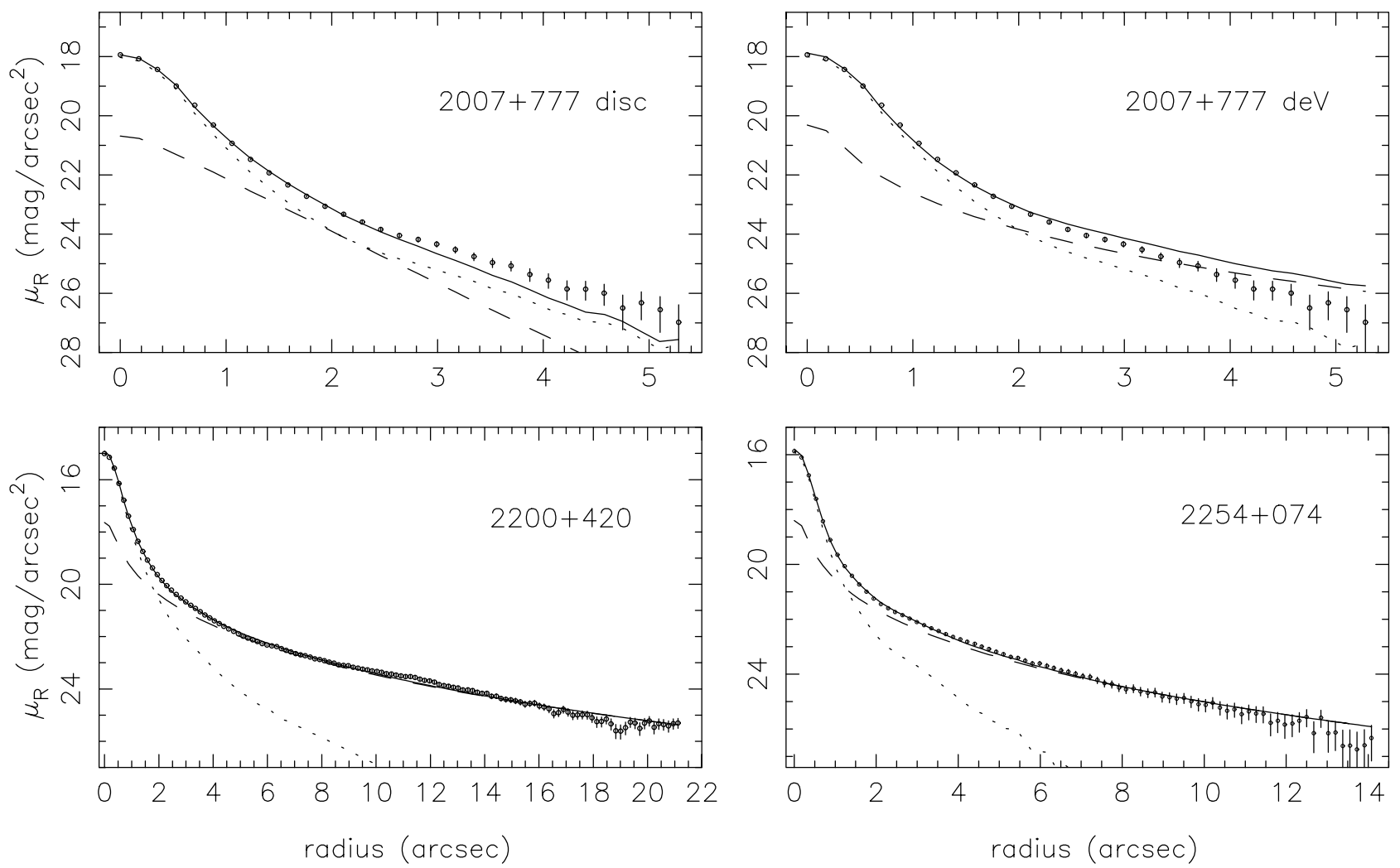

Fig. 1. continued.

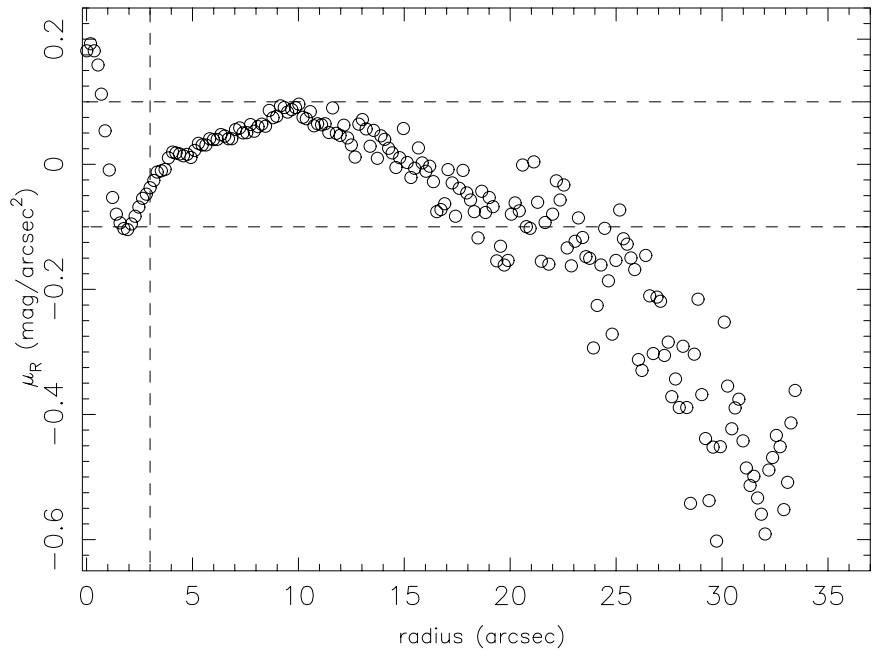

Fig. 2. The residuals (observed - model) of the best fit de Vaucouleurs-model for $1807+698$. The vertical line shows the limit when the PSF is about 2 mag fainter than the galaxy. The horizontal lines show the \pm 0.1 mag residuals.

In comparison to A91 and Wurtz et al. (1996 hereafter W96), the trend is that using the deeper NOT data, the derived parameters suggest larger host galaxies. A91 got slightly fainter magnitudes but the results of W96 differ between \pm 0.3 . Also in contradiction to W96, deeper NOT images revealed no sign of the host galaxy in $0814+425$ and $0823+033$ (see Appendix A). The seeing was somewhat poorer in the W96 data and also the integration time was shorter (average $\sim 750 \mathrm{~s}$ ). On average S93 derived somewhat larger and fainter host galaxies than the present work. This could be due to the different analysing method and also because the seeing was somewhat poorer. S93 excluded the innermost $5^{\prime \prime}$ from the fit and the fitting procedure was two-dimensional. Interestingly, using the same data for $1652+398$, as in this study, two-dimensional fitting (N99) gave a slightly fainter and smaller host galaxy than this study. This suggests that due to the different analysing procedure $\Delta m$ could be $0.2 \mathrm{mag}$ and $\Delta r_{\mathrm{e}} \sim 20 \%$, which are in accordance with the findings of SU00. A more detailed comparison can be found in Appendix B. The faintest (marginally) detected host galaxy has $m_{R} \sim 20$ mag. Taking into account the higher average redshift of the objects, our host galaxy detection rate is similar to FK99.

\subsection{Comparison with the HST data}

The recent Hubble Space Telescope (HST) snapshot survey presented short (average $\sim 10 \mathrm{~min}$ ) exposures of 110 BL Lac objects (U00, SU00). Figure 4 compares the galaxy parameters derived in U00 and the present work. The agreement is very good, yet there is a small systematic difference in the galaxy magnitudes ( $m_{\text {host }}$ is slightly brighter in the NOT data). The largest differences are in the nearby well-resolved objects (AP Lib, 3C371, BL Lac). These differences could arise from the short exposure times of the HST data. FK99 compared the HST snapshot 

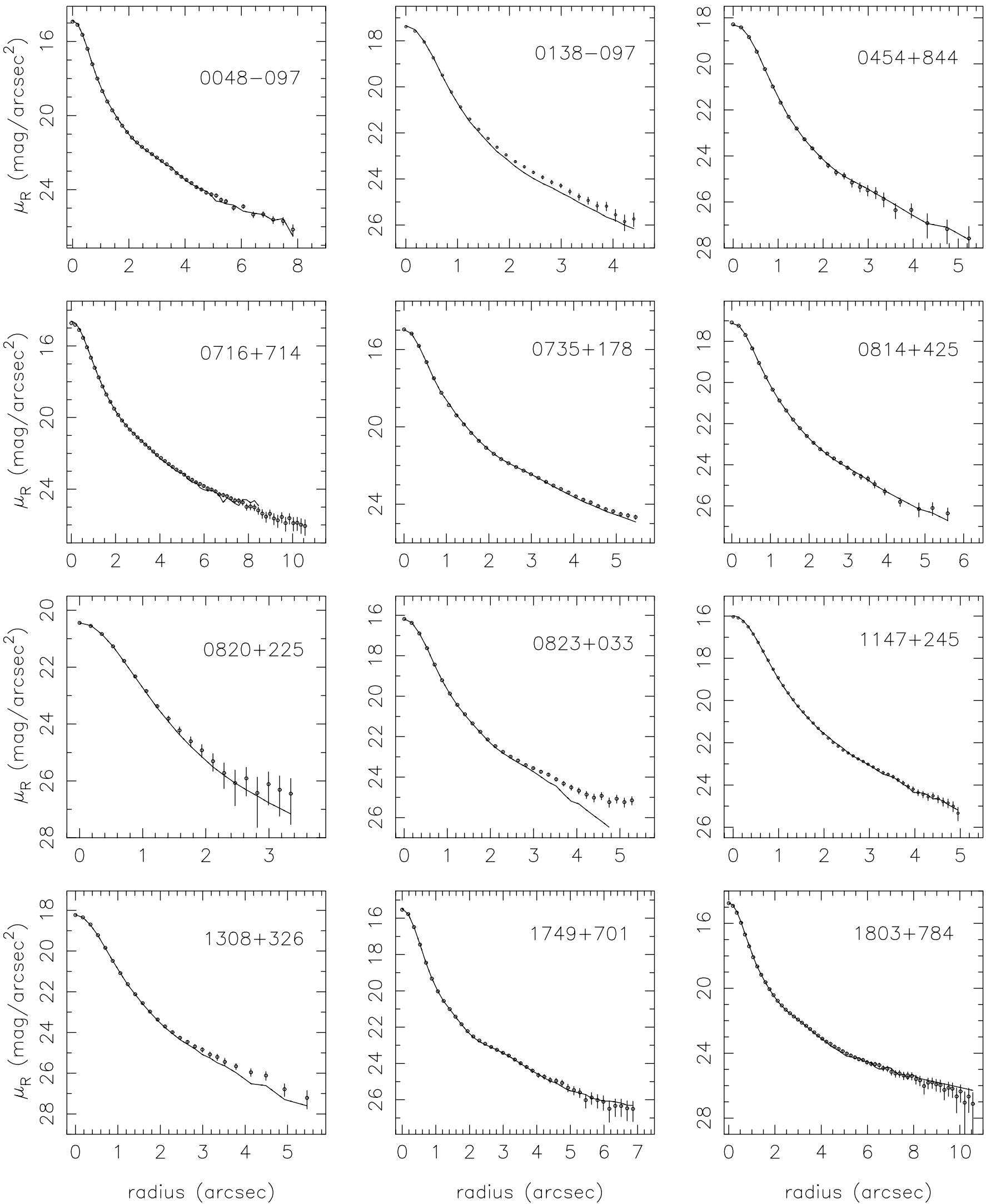

Fig. 3. The observed azimuthally averaged radial profiles of the unresolved $1 \mathrm{Jy}$ BL Lac objects. Filled dots represents the observed profile and line the best fit PSF. 

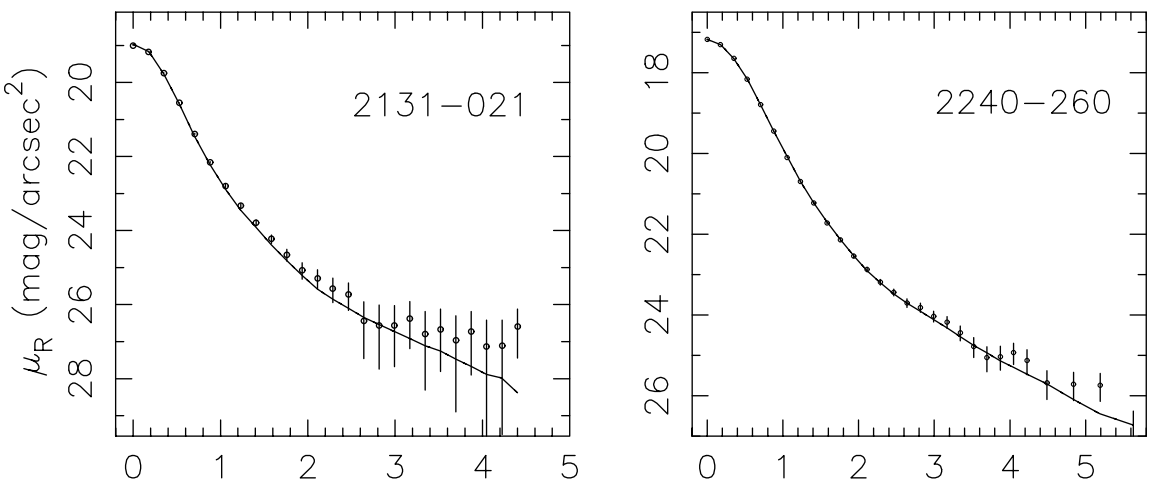

Fig. 3. continued.

Table 3. Unresolved objects. For each object the first row gives only the best point source fit and the second de Vaucouleurs model with a fixed $r_{\mathrm{e}}=1^{\prime \prime}$. A colon after the point source magnitude denotes objects for which PSF has not been constructed from the same field as the object.

\begin{tabular}{llcccr}
\hline Object & $m_{\mathrm{AGN}}$ & err & & & $\chi^{2} /$ d.o.f. \\
& & & $m_{\mathrm{host}}^{\mathrm{deV}}$ & $\left.r_{\mathrm{e}},{ }^{\prime \prime}\right]$ & $\chi^{2} /$ d.o.f. \\
\hline $0048-097$ & 15.1 & 0.1 & $\ldots$ & $\ldots$ & 0.99 \\
& 15.2 & 0.1 & 18.3 & 1.0 & 2.51 \\
$0138-098$ & $17.43:$ & 0.01 & $\ldots$ & $\ldots$ & 19.64 \\
& $17.55:$ & 0.01 & $19.5:$ & 1.0 & 1.61 \\
$0454+844$ & 18.22 & 0.01 & $\ldots$ & $\ldots$ & 3.36 \\
& 18.30 & 0.01 & 20.6 & 1.0 & 9.24 \\
$0716+714$ & $14.11:$ & 0.01 & $\ldots$ & $\ldots$ & 2.17 \\
& $14.34:$ & 0.01 & $16.7:$ & 1.0 & 3.76 \\
$0735+178$ & 15.26 & 0.01 & $\ldots$ & $\ldots$ & 1.29 \\
& 15.36 & 0.01 & 18.6 & 1.0 & 3.06 \\
$0814+425$ & 17.01 & 0.01 & $\ldots$ & $\ldots$ & 6.46 \\
& 17.10 & 0.01 & 19.5 & 1.0 & 16.79 \\
$0820+255$ & 19.89 & 0.01 & $\ldots$ & $\ldots$ & 0.61 \\
& 20.00 & 0.01 & 22.0 & 1.0 & 1.64 \\
$0823+033$ & 16.35 & 0.01 & $\ldots$ & $\ldots$ & 7.26 \\
$1147+245$ & $15.87:$ & 0.01 & $\ldots$ & $\ldots$ & 3.86 \\
& $15.92:$ & 0.01 & $19.4:$ & 1.0 & 7.98 \\
$1308+326$ & 17.92 & 0.01 & $\ldots$ & $\ldots$ & 2.10 \\
& 18.03 & 0.01 & 20.0 & 1.0 & 5.51 \\
$1749+701$ & 16.06 & 0.01 & $\ldots$ & $\ldots$ & 0.49 \\
& 16.13 & 0.01 & 19.7 & 1.0 & 1.17 \\
$1803+784$ & 14.65 & 0.01 & $\ldots$ & $\ldots$ & 0.94 \\
& 14.75 & 0.01 & 18.0 & 1.0 & 1.67 \\
$2131-021$ & 19.27 & 0.01 & $\ldots$ & $\ldots$ & 4.32 \\
& 19.40 & 0.01 & 20.8 & 1.0 & 10.92 \\
$2240-260$ & 16.89 & 0.01 & $\ldots$ & $\ldots$ & 0.50 \\
& 16.92 & 0.01 & 20.4 & 1.0 & 1.24 \\
\hline & & & & &
\end{tabular}

and their NOT data and found very good agreement between the two data sets. Their conclusion was that ideally one should combine the data using the core $\left(r \lesssim 1-3^{\prime \prime}\right)$ of the high resolution HST image and the wings from deep ground based (e.g. NOT) images.

\subsection{Host galaxies}

Table 5 shows the absolute values of the nucleus and host galaxy brightness and the size of $1 \mathrm{Jy}$ BL Lac objects.
Table 4. Observed host galaxy properties for 1 Jy BL Lac objects from various ground-based optical imaging studies. The $r$-magnitudes have been corrected by 0.3 (Fukugita et al. 1995).

\begin{tabular}{|c|c|c|c|c|c|}
\hline Object & galaxy & $r_{\mathrm{e}}\left[^{[\prime \prime}\right]$ & ref. & type & Comm. \\
\hline \multirow[t]{4}{*}{$1418+546$} & 16.2 & 3.5 & S93 & $\mathrm{deV}$ & $2 \mathrm{D}$ \\
\hline & 16.4 & 2.64 & A91 & disc & \\
\hline & 16.61 & 2.4 & W96 & disc & $r$-band \\
\hline & 16.04 & 2.32 & this & $\mathrm{deV}$ & \\
\hline \multirow[t]{3}{*}{$1514-241$} & 14.4 & 8.7 & S93 & $\mathrm{deV}$ & $2 \mathrm{D}$ \\
\hline & 14.6 & 5.66 & A91 & $\mathrm{deV}$ & \\
\hline & 14.29 & 6.72 & this & $\mathrm{deV}$ & \\
\hline \multirow[t]{2}{*}{$1538+149$} & 19.59 & 1.5 & W96 & $\operatorname{deV}(?)$ & $r$-band \\
\hline & 19.86 & 3.99 & this & $\mathrm{deV}$ & \\
\hline \multirow[t]{5}{*}{$1652+398$} & 12.8 & 20.0 & S93 & $\mathrm{deV}$ & $2 \mathrm{D}$ \\
\hline & 12.7 & 9.3 & A91 & $\mathrm{deV}$ & \\
\hline & 12.69 & 9.0 & W96 & $\mathrm{deV}$ & $r$-band \\
\hline & 12.60 & 13.0 & N99 & $\mathrm{deV}$ & $2 \mathrm{D}$ \\
\hline & 12.42 & 17.21 & this & $\mathrm{deV}$ & \\
\hline \multirow[t]{2}{*}{$1749+096$} & 17.73 & 7.1 & W96 & $\mathrm{deV}$ & $r$-band \\
\hline & 18.70 & 1.35 & this & $\mathrm{deV}$ & \\
\hline \multirow[t]{3}{*}{$1807+698$} & 14.4 & 14.9 & $\mathrm{~S} 93$ & $\mathrm{deV}$ & $2 \mathrm{D}$ \\
\hline & 13.82 & 10.5 & W96 & $\mathrm{deV}$ & $r$-band \\
\hline & 14.17 & 10.76 & this & $\mathrm{deV}$ & \\
\hline \multirow[t]{2}{*}{$2007+777$} & 18.90 & 2.1 & W96 & $\operatorname{deV}(?)$ & $r$-band \\
\hline & 18.89 & 2.89 & this & $\operatorname{deV}(?)$ & \\
\hline \multirow[t]{3}{*}{$2200+422$} & 15.3 & 5.8 & S93 & $\mathrm{deV}$ & $2 \mathrm{D}$ \\
\hline & 14.57 & 10.2 & W96 & $\mathrm{deV}$ & $r$-band \\
\hline & 15.00 & 6.63 & this & $\mathrm{deV}$ & \\
\hline \multirow[t]{4}{*}{$2254+074$} & 16.6 & 3.4 & S93 & $\mathrm{deV}$ & 2D \\
\hline & 16.45 & 4.1 & W96 & $\mathrm{deV}$ & $r$-band \\
\hline & 16.8 & 3.5 & F96 & $\mathrm{deV}$ & \\
\hline & 16.52 & 4.57 & this & $\mathrm{deV}$ & \\
\hline
\end{tabular}

The median absolute magnitude of the eleven resolved objects is $-23.82 \pm 0.60$ (the average being -24.05 ). The host galaxy magnitudes from different studies are compared in Table 6. Generally all the results agree well with each other and also these values agree well with the host galaxies of $z \leq 0.12$ radio galaxies by Govoni et al. (2000). Figure 5 shows the magnitude distributions of the $1 \mathrm{Jy}$ and EMSS/Slew samples, which are very similar to each other. Using the Kolmogorov-Smirnov (KS) test the probability that the two distributions are drawn from the same population is $38 \%$. It should be noted that 
Table 5. Absolute values of the host galaxies. All the values are derived assuming de Vaucouleurs profile and assuming $r_{\mathrm{e}}=1^{\prime \prime}$. 0 for unresolved objects. In the last column U stands for unresolved, $\mathrm{M}$ for marginally resolved, and $\mathrm{E}$ for an elliptical type host galaxy. A question mark is shown when the morphology of the host galaxy remains uncertain.

\begin{tabular}{lrrcl}
\hline Name & $M_{\mathrm{AGN}}$ & $M_{\text {host }}$ & $r_{\mathrm{e}}[\mathrm{kpc}]$ & Comm. \\
\hline $0138-098$ & -26.48 & $>-26.0$ & $\ldots$ & $\mathrm{U}$ \\
$0235+164$ & -26.10 & $\ldots$ & & $\mathrm{N} 96$ \\
$0454+844$ & -27.62 & $>-28.4$ & $\ldots$ & $\mathrm{U} z_{\mathrm{abs}}$ \\
$0735+178$ & -27.30 & $>-24.6$ & $\ldots$ & $\mathrm{U} z_{\mathrm{abs}}$ \\
$0814+425$ & -28.55 & $>-29.0$ & $\ldots$ & $\mathrm{U}$ \\
$0820+225$ & -24.82 & $>-24.9$ & $\ldots$ & $\mathrm{U}$ \\
$0823+033$ & -26.72 & & & \\
$0851+202$ & -26.44 & -23.18 & $6.0 \pm 1.7$ & $\mathrm{M}$ \\
$1308+326$ & -26.78 & $>-27.0$ & $\ldots$ & $\mathrm{U}$ \\
$1418+546$ & -24.31 & -24.11 & $8.3 \pm 0.2$ & $\mathrm{E}$ \\
$1514-241$ & -22.23 & -23.55 & $8.9 \pm 0.1$ & $\mathrm{E}$ \\
$1538+149$ & -25.97 & -24.70 & $35.5 \pm 18.8$ & $\mathrm{E}$ \\
$1652+398$ & -22.51 & -24.20 & $15.7 \pm 0.2$ & $\mathrm{E}$ \\
$1749+096$ & -22.70 & -23.82 & $8.4 \pm 0.7$ & $\mathrm{E} ?$ \\
$1749+701$ & -28.10 & $>-26.1$ & $\ldots$ & $\mathrm{U}$ \\
$1803+784$ & -29.15 & $>-27.2$ & $\ldots$ & $\mathrm{U}$ \\
$1807+698$ & -23.00 & -23.57 & $14.8 \pm 0.2$ & $\mathrm{E}$ \\
$1823+568$ & -25.72 & -25.30 & $24.3 \pm 5.3$ & $\mathrm{E} ?$ \\
$2007+777$ & -24.42 & -23.80 & $18.7 \pm 1.9$ & $\mathrm{E} ?$ \\
$2131-021$ & -26.31 & $>-27.8$ & $\ldots$ & $\mathrm{U}$ \\
$2200+420$ & -24.09 & -23.77 & $12.0 \pm 0.4$ & $\mathrm{E}$ \\
$2240-260$ & -27.20 & $>-25.3$ & $\ldots$ & $\mathrm{U}$ \\
$2254+074$ & -24.35 & -24.40 & $19.5 \pm 1.1$ & $\mathrm{E}$ \\
\hline
\end{tabular}

Table 6. Host galaxy properties. Median of the $M_{\text {host }}$ and $r_{\mathrm{e}}$ with standard deviations. The HST pointed observations (Urry et al. 1999, hereafter U99) $I$-band magnitudes are corrected using $R-I$-colours for E galaxies (Fukugita et al. 1995). The W96 host $r$-band magnitudes have been transformed to $R$-band assuming $R-r=0.3$ and the applied correction for the different cosmology is $0.1 \mathrm{mag}$. Similarly scale lengths have been corrected to $q_{0}=0.0$ cosmology

\begin{tabular}{lrcrl}
\hline Sample & $n$ & $\left\langle M_{\text {host }}\right\rangle$ & \multicolumn{1}{c}{$\left\langle r_{\mathrm{e}}\right\rangle$} & Comm. \\
\hline S93 & 7 & $-23.90 \pm 0.32$ & $12.4 \pm 5.1$ & 1 Jy \\
W96 & 41 & $-23.7 \pm 0.7$ & $10.3 \pm 8.3$ & $z<0.5$ \\
U99 & 7 & $-23.85 \pm 0.54$ & $9.3 \pm 6.5$ & HST deep \\
FK99 & 42 & $-23.92 \pm 0.59$ & $7.9 \pm 4.9$ & EMSS/Slew \\
U00 & 63 & $-23.71 \pm 0.56$ & $8.5 \pm 5.6$ & HST snap \\
This & 11 & $-23.82 \pm 0.60$ & $14.8 \pm 8.7$ & 1 Jy \\
\hline Govoni et al. & 79 & $-24.11 \pm 0.69$ & $14.0 \pm 9.7$ & RG \\
\hline
\end{tabular}

almost half of the host galaxy magnitudes have only upper limits (Table 5). The mean for the whole sample was estimated using Astronomy SURVival analysis software (ASURV; Feigelson \& Nelson 1985 and Isobe et al. 1986) installed in IRAF/STSDAS. For all objects with known redshift, using the Kaplan-Meier estimator for the mean is $\left\langle M_{R}\right\rangle=-23.70 \pm 0.20$.

The observed scale length values are between 6.0 and $35.5 \mathrm{kpc}$, however the largest values have the largest uncertainties as well (Table 5). The median $r_{\mathrm{e}}$ is $14.8 \pm 8.7 \mathrm{kpc}$ without signs of resdshift dependence. This is a slightly
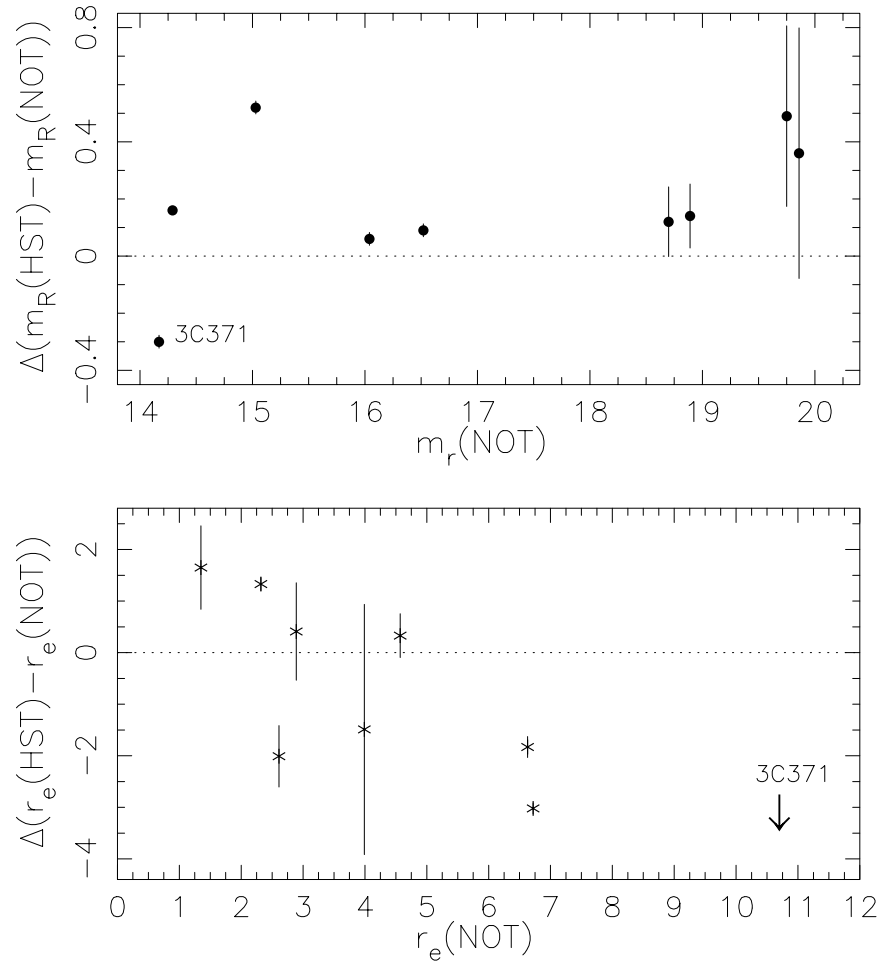

Fig. 4. Comparison of the present work and HST snap shot (U00). For 3C371, SU00 derived $r_{\mathrm{e}}=2^{\prime \prime}$. 1 .

larger value than FK99 or U00 found, but within the errors (Table 6). The small offset between the RBLs and $\mathrm{XBLs}$ is most likely due to the deeper images of the RBL sample, because U00 found no difference between the scale lengths of RBLs and XBLs. In comparison to radio galaxies these results agree very well. These results suggest that the host galaxies of RBLs and XBLs have similar sizes and the hosts of FR I/II radio galaxies are similar to the host galaxies of BL Lac objects, at least at low redshifts.

Figure 6 shows the $M_{\text {host }}$ vs. $\log z$ plane for BL Lac objects. Again the $1 \mathrm{Jy}$ objects fall well into the EMSS/Slew distribution without any signs of bimodality. Also in comparison to the complete EMSS (C-EMSS) sample (Morris et al. 1991) the distributions are similar. For the high- $z$ objects the derived upper limits are well above the evolutionary tracks for a $M^{*}-1$ galaxy as predicted by Bressan et al. (1994). The two $z \sim 0.6$ host galaxies support the tentative positive correlation of host luminosity with redshift suggested by FK99. Even deeper images of distant $(z \gtrsim 0.6)$ objects are needed for the galaxy evolution studies.

Above, it was found that 1 Jy host galaxies have similar brightness and size distributions to the EMSS/Slew sample. To study the morphology of the host galaxies a more detailed comparison is needed. Elliptical galaxies populate a thin plane (Fundamental Plane, FP), which is defined by the effective radius $r_{\mathrm{e}}$, the effective mean surface brightness $\mu_{\mathrm{e}}$ and the velocity dispersion $\sigma$ (Djorgovski \& Davis 1987). Without the information of $\sigma$, studies were made using the $\mu_{\mathrm{e}}-r_{\mathrm{e}}$ projection 


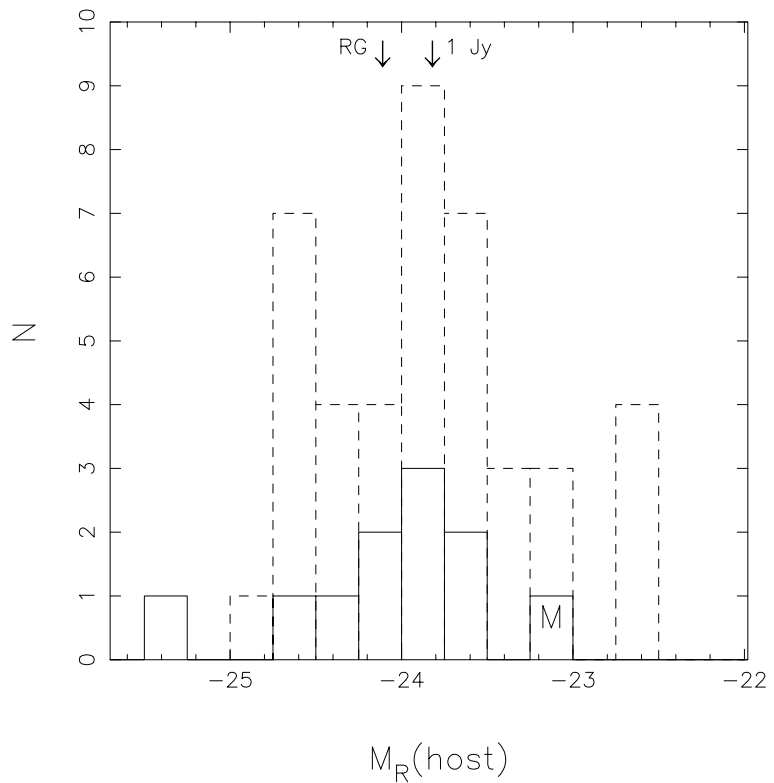

Fig. 5. The distribution of the absolute magnitude of the host galaxies of BL Lac objects. The solid line corresponds to objects from 1 Jy sample and the dashed line EMSS and Slew survey objects from FK99. The arrows indicate the median value of the 1 Jy sample and radio galaxies from Govoni et al. (2000) sample. Marginal detection of the host galaxy of $0851+202$ is marked with the letter $\mathrm{M}$.

of the FP (Kormendy relation; Kormendy 1977). The observed $\mu_{\mathrm{e}}$ from the fit was corrected for Galactic absorption, $K$-correction and for the cosmological $(1+z)^{4}$ dimming. The best fit for all (11) resolved ${ }^{3}$ objects is $\mu=17.85 \pm 0.68+3.27 \pm 0.59 \times \log \left(r_{\mathrm{e}}\right)$ which is very close to the findings of FK99 and U00; their best fits are $\mu=16.45+4.6 \times \log \left(r_{\mathrm{e}}\right)$ and $\mu=17.2+3.9 \times \log \left(r_{\mathrm{e}}\right)$, respectively. The host galaxies from the 1 Jy sample follow the same trend as EMSS/Slew samples and are indistinguishable in the $\mu-r_{\mathrm{e}}$-plane (Fig. 7). This confirms the results by FK99 and U00 that BL Lac hosts are luminous elliptical galaxies, about 1 mag brighter than $M^{*}$.

The similarity between the host galaxies of RBLs and XBLs is striking. All of the above comparisons $\left(M_{\text {host }}, r_{\mathrm{e}}\right.$ and Kormendy relation) suggest that the host galaxies of the $1 \mathrm{Jy}$ sample and EMSS/Slew sample are similar. This indicates that the relationship between the type of activity (e.g. the peak frequency of the synchrotron component) and the type of host galaxy is weak or does not exist. This supports the idea that BL Lacs belong to one family of objects (at least $z \lesssim 0.5$ objects) of which LBLs and HBLs are the extreme ends. These results support the $M-z$ trend found by W96 and FK99, and also agree with their conclusion that low- $z$ BL Lacs are drawn from the population of giant ellipticals rather than from field ellipticals.

${ }^{3}$ If three objects with large errors are excluded the best fit is $\mu=16.66 \pm 0.95+4.41 \pm 0.85 \times \log \left(r_{\mathrm{e}}\right)$.

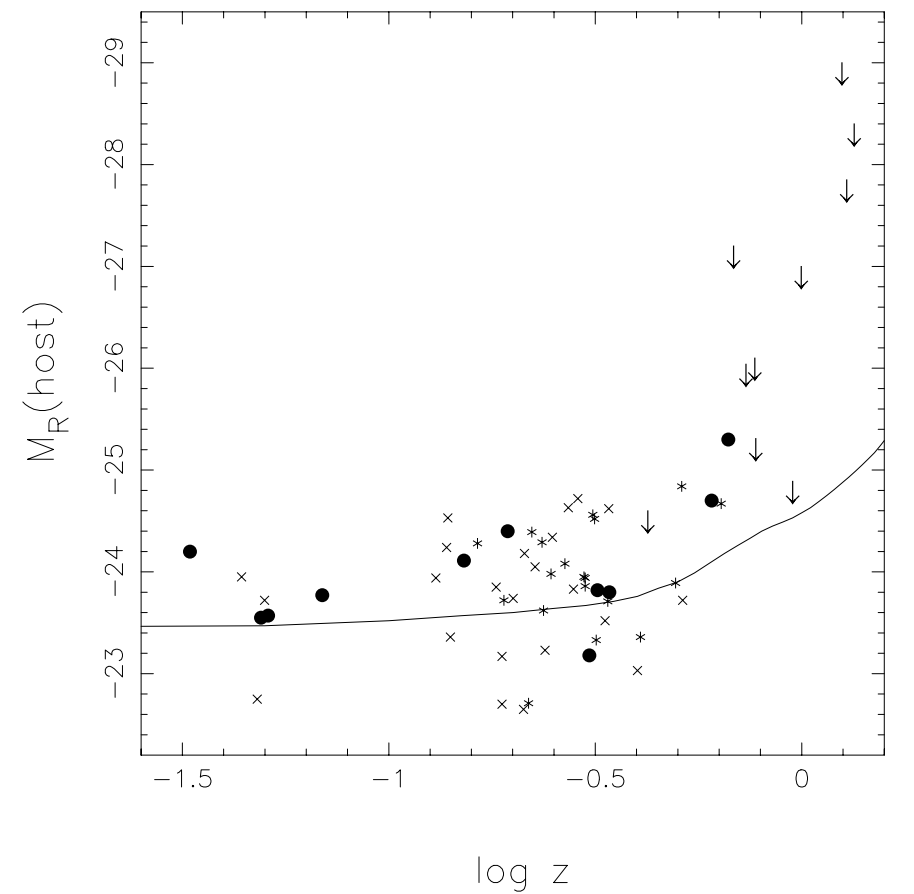

Fig. 6. The absolute brightness of the host galaxy versus redshift. Resolved 1 Jy objects are indicated with filled dots and upper limits with an arrow. The results of FK99 are shown with stars (C-EMSS sample) and crosses. The solid line corresponds to a passively evolving $M^{*}-1$ mag galaxy (Bressan et al. 1994).

\subsection{Properties of the nuclear source}

The active nucleus to starlight ratio $\left(\log \left(L_{\mathrm{AGN}} / L_{\mathrm{host}}\right)\right)$ is shown in Fig. 8 for the $1 \mathrm{Jy}$ and EMSS/Slew samples. For the resolved objects, $\log \left(L_{\mathrm{AGN}} / L_{\text {host }}\right)$ varies from -0.6 to 1.3 with an average of $0.05 \pm 0.55$ (median 0.08 ). For the whole sample the Kaplan-Meier estimated average is $0.52 \pm 0.17$. The $\log \left(L_{\mathrm{AGN}} / L_{\text {host }}\right)$ distribution of the 1 Jy objects is similar to the FK99 objects. However, the most AGN dominated sources are LBLs and the most host dominated objects are HBLs. The lack of LBLs in the lower part of Fig. 8 (i.e. the most negative ratios) is possibly due to the selection criteria of the $1 \mathrm{Jy}$ sample. The requirement of a featureless spectrum criterium rejects not only objects with strong emission lines but also objects which have either relatively weak emission from the nucleus or a very powerful host galaxy (i.e. small $\log \left(L_{\mathrm{AGN}} / L_{\mathrm{host}}\right)$-ratio $)$. This selection bias has already been noted by Browne \& Marchã (1993), the so-called Browne-Marchã effect.

The correlation between $M_{\mathrm{AGN}}$ and redshift is shown in Fig. 9. The median $M_{\mathrm{AGN}}$ for the whole sample is $-26.10 \pm 1.97$ (21 objects, including AO 0235+164 and excluding objects with absorption line redshifts). The dashed line in Fig. 9 shows the absolute brightness of a 20 mag point source at different distances, which is roughly the limiting magnitude of the POSS-plates from which the objects were initially identified. Note that almost all powerful objects are high- $z$ LBLs and also that the most distant 


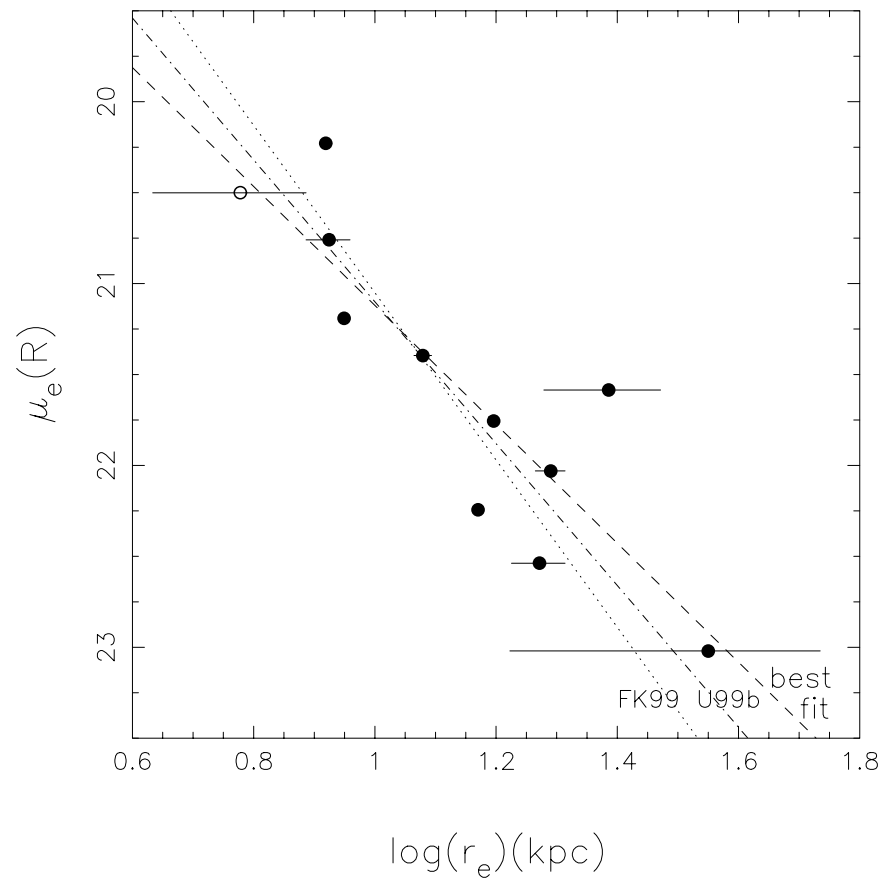

Fig. 7. The Kormendy relation. The best fit of the $\mu_{\mathrm{e}}-\log (r)$ for the current sample is indicated by a dashed line. The dotted and dash-dotted lines show the results by FK99 and U00, respectively. A marginally resolved object (OJ287) is shown with an open symbol.

EMSS objects $(1517+656$, Beckmann et al. 1999) fall in to the 1 Jy objects distribution. In the 1 Jy sample there is a strong apparent correlation between the brightness of the nucleus and redshift. Dividing the sample based on the emission line redshift, the median is $-24.1 \pm 1.32$ for objects with $z<0.5$ (9 objects) and $-26.6 \pm 1.24 z>0.5$ objects (12 objects). Most likely this arises from the sample's selection criteria.

The brightness distribution of the $z \lesssim 0.51 \mathrm{Jy}$ objects is similar to the EMSS/Slew objects (the average is $-22.97 \pm 1.61$; all 43 objects, $-22.89 \pm 1.46$; $z<0.5,39$ objects). The KS-probability that the two samples are drawn from the same parent population is $P_{\mathrm{KS}}=83 \%$. However, incomplete redshift information causes some unknown biases in the $1 \mathrm{Jy}$ sample. The shapes of the SEDs are the same for both HBLs and LBLs, but Fossati et al. (1998) found that LBLs have more luminous jets than HBLs. If this is the case, the same optical brightness distribution is due to a coincidence. As the peak frequency of the SED increases, the spectra of the two types of objects (LBL and HBL) must intersect somewhere between IR and X-ray (in $\log (S), \log (\nu)$-plane).

In recent studies of QSOs and AGNs it has been proposed that there is a correlation between the absolute brightness of the AGN and the host galaxy (McLure et al. 1999; Schade et al. 2000). This could reflect the accretion rate to the black hole. However, in some other studies such a correlation has not been found (Govoni et al. 2000). For BL Lac objects FK99 found an indication of a correlation, but none was found for U00.

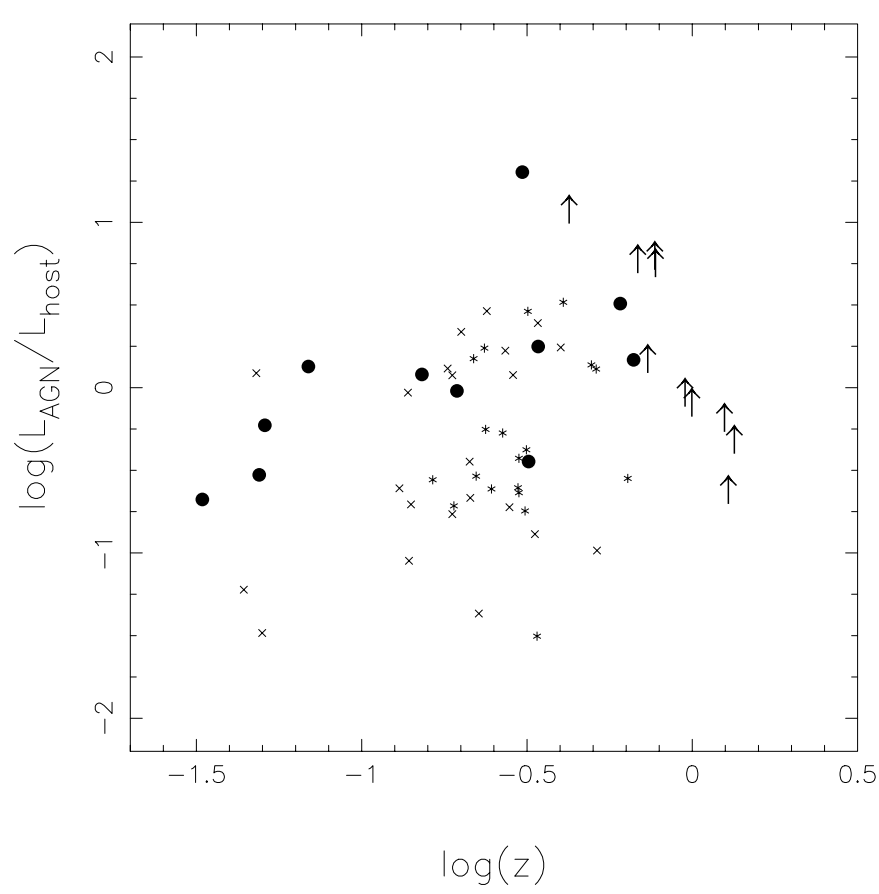

Fig. 8. Light ratio $\log \left(L_{\mathrm{AGN}} / L_{\text {host }}\right)$ vs. $\log (z)$. The symbols are the same as in Fig. 6.

Figure 10 shows the results of the correlation between the nucleus and the host galaxy of $1 \mathrm{Jy}$ and EMSS/Slew samples. There is an indication of a trend similar to FK99's findings, however, the statistical significance of it is less than $2 \sigma$. The slope $(\sim 0.1)$ of the possible correlation is more gentle than e.g. that found by Schade et al. (2000). The trend might be due to selection effects, e.g. lack of 1 Jy objects in the upper left hand corner in a similar way to Fig. 8 above. Figures 9 and 10 suggests that for objects with $z \lesssim 0.5$ or $M_{\mathrm{AGN}} \gtrsim-25$ there is no strong nucleus-galaxy correlation. This is in accordance with the lack of correlation in the Govoni et al. (2000) radio galaxy sample.

\section{Summary}

In the present paper, the optical ( $R$-band) properties of the 1 Jy sample BL Lacs have been studied. Out of 25 observed BL Lacs (a total sample of 37) 11 were resolved. In well resolved objects (six low- $z$ ) an elliptical galaxy was preferred and of the remaining cases, only once did a disc-type galaxy give gave better fit, however in this case neither can be preferred. In comparison to X-ray selected BL Lacs (or HBLs) the galaxy properties were indistinguishable ( $M_{\text {host }}, r_{\mathrm{e}}$ and Kormendy relation). This suggests that BL Lacs (HBLs and LBLs at low- $z$ ) belong to one group of objects. Also the host galaxy properties are similar to radio galaxies, which supports the unification schemes of radio-loud AGNs (i.e. BL Lacs are beamed radio galaxies). These results confirm the recent findings (e.g. host galaxy properties, similarity between HBLs and LBLs) of FK99 and U00. 


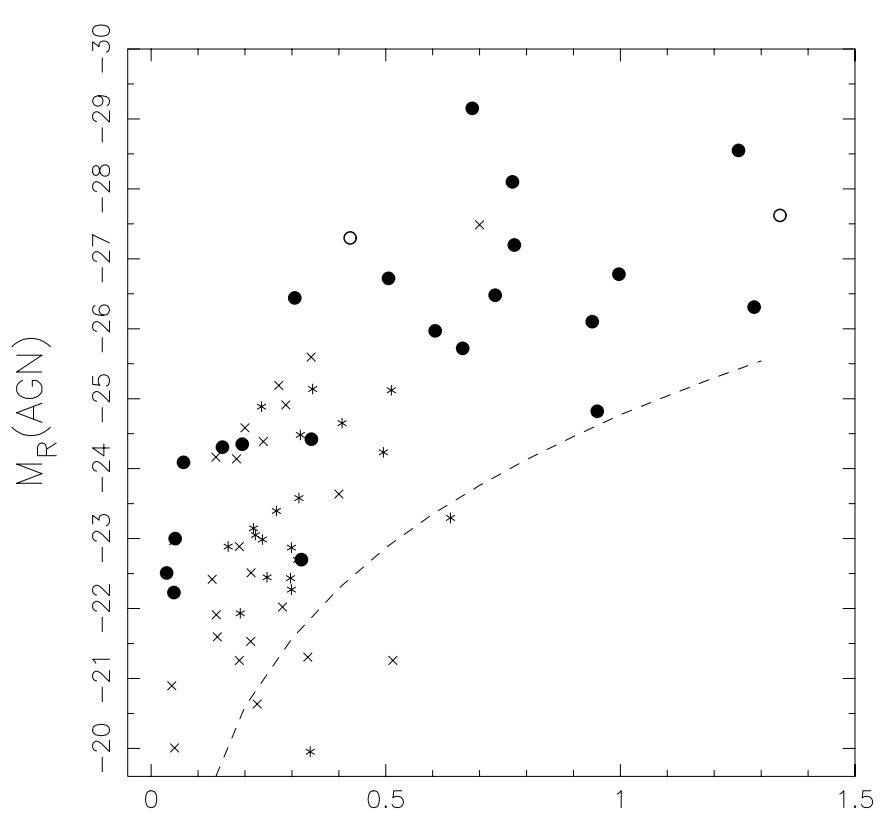

Z

Fig. 9. The correlation with the absolute brightness of the nuclear component and redshift. The symbols are the same as in Fig. 6. The objects with absorption line redshift are marked with an open circle. The dashed line corresponds to a 20 mag object at different redshifts.

There is a strong correlation between the brightness of the nucleus and redshift. The absolute brightness of the nuclear component of LBLs is similar to the HBLs. Also HBLs and LBLs are indistinguishable in the nucleus/host galaxy light ratio distribution. Finally the nuclear brightness is almost independent of the type of BL Lac (at $z \lesssim 0.5)$ and the host galaxy brightness.

Acknowledgements. The NASA/IPAC Extragalactic Database (NED) is operated by the Jet Propulsion Laboratory, California Institute of Technology, under contract with the National Aeronautics and Space Administration. Thanks to Riccardo Scarpa for the use of the PROFIT package. This work was partly supported by the Academy of Finland. JH acknowledges the support of the DFG through SFB 328 and TP acknowledges the support of the Väisälä Foundation.

\section{Appendix A: Notes on individual objects}

0048-097 This unresolved object is without known redshift. Previously F96 and S93 reported this object to be unresolved. Calibration of the NOT data is uncertain.

0138-097 The core of the PSF was from a field star $\left(m_{R} \sim 19\right)$ and the wings from a star observed during the same observing run. The southern companions (B and C in Heidt et al. 1996) were masked out from the fitting. The best fit de Vaucouleurs model gives $m_{\text {host }}=19.43$, $r_{\mathrm{e}}=0$ ". $82\left(M_{\text {host }}=-26.06\right.$ and $\left.r_{\mathrm{e}}=8 \mathrm{kpc}\right)$ with $\chi^{2}=0.51$, which is a lower value than for a pure point source (Table 3). However, the data do not fit the FP in Fig. 7 (2.5 mag above the best fit), hence this object is

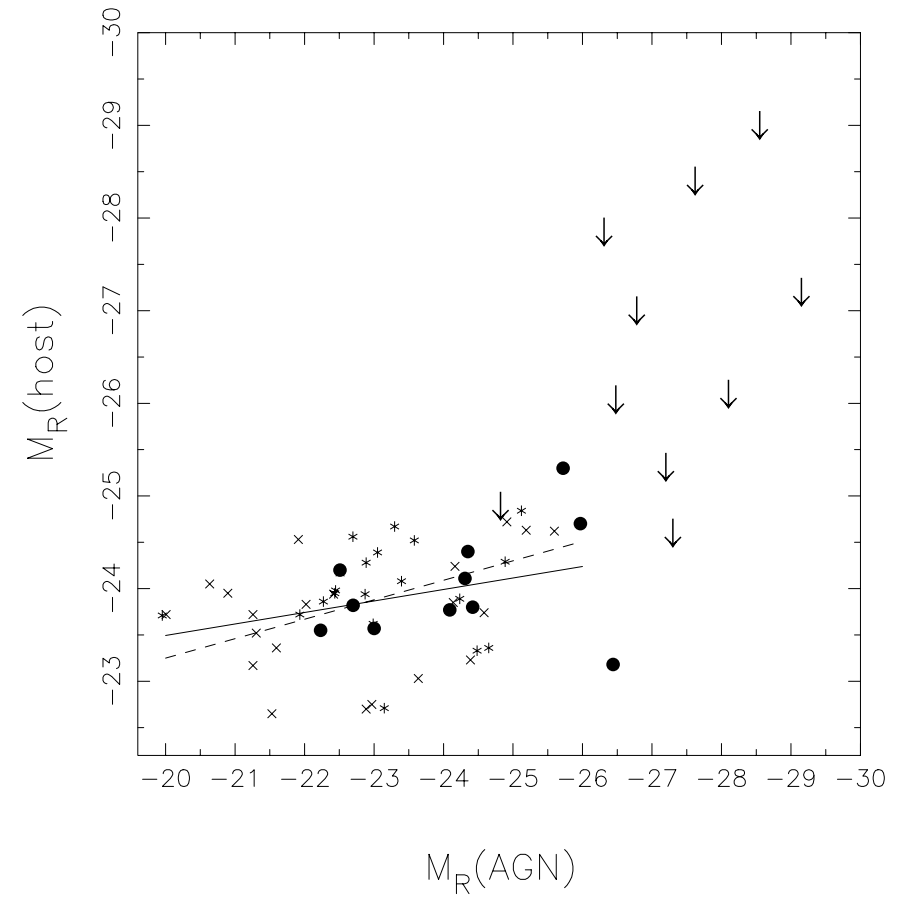

Fig. 10. Absolute brightness of the host galaxy and the nucleus. The best fit regression of the resolved objects is marked with a solid line (slope 0.12 ). The dashed line shows the correlation between the host galaxy and the nuclear absolute magnitude (slope 0.21) for X-ray selected AGN found by Schade et al. (2000).

judged as unresolved. SU00 found this object to be unresolved, yet with extra emission at large radii. They proposed that the excess would be from the nearby companion galaxies.

$\mathbf{0 4 5 4}+\mathbf{8 4 4}$ The radial profile matches perfectly with the PSF and there are no indications of the host galaxy. This is in accordance with the results of S93 and SU00 and also with the high redshift $(z=1.340)$.

$\mathbf{0 7 1 6}+\mathbf{7 1 4}$ Redshift of this object is unknown. The PSF was constructed using the core from a faint star $\left(m_{R} \sim\right.$ 19.5) in the same field as the object and wings from a star during the same night observed with a similar seeing. Seeing was variable during these observations. In S93, SU00 and in the present NOT data observations this object remained unresolved which suggests that this object is distant.

$\mathbf{0 7 3 5}+\mathbf{1 7 8}$ The redshift $(z=0.424)$ of this object is based on a MgII absorption line. The star $\sim 10^{\prime \prime}$ to the east was subtracted and the companion galaxy ( $\sim 9^{\prime \prime}$ north-west) was masked. Despite the deep image with an excellent seeing, no signs of the host galaxy were found. This object has also remained unresolved in previous observations (A91, S93, SU00). The nearby environment of this object has been shown in Pursimo et al. (1999).

0814+425 Contrary to W96 results, this object remains unresolved, which is similar to the results of S93 and Falomo et al. (1997a). The redshift of this object is 
uncertain (Lawrence et al. 1996), however these results suggest a large distance $z \gtrsim 0.3$ (cf. 0851+202).

$\mathbf{0 8 2 0}+\mathbf{2 5 5}$ No indications of the host galaxy were found, in accordance with S93 and SU00.

0823+033 A star (4". 4 east) with similar brightness to the object and faint objects within a $5^{\prime \prime}$ radius, make the tail of the profile uncertain. Before extracting the radial profile, the star was subtracted using a scaled PSF from the same field and the companion was masked. The pure PSF fits the profile perfectly up to $\sim 2$.' 5 . The nature of the excess in the wings of the profile is uncertain (Fig. 3), which could be due to the improper masking and subtraction of the star. Due to light contamination, it is impossible to estimate the minimum brightness of the host galaxy. S93 reported this object resolved and W96 marginally resolved, when the object was about one magnitude fainter than in the present data. However, SU00 interpreted the excess light they found as not originating from the host galaxy.

$\mathbf{0 8 5 1 + 2 0 2}$ This object (OJ287) is already reported to have been marginally resolved by Hutchings et al. (1984) and later S93 also claimed it to be marginally resolved. W96 reported it marginally resolved and Yanny et al. (1997) confirmed this. In the NIR observations, Wright et al. (1998) found OJ287 resolved, but not in their optical images. In the recent $H S T$ snapshot image (5 min exposure) OJ287 remains unresolved (SU00), yet it is found resolved in the VLT image (Heidt et al. 1999b) with $m_{R}=18.41, r_{\mathrm{e}}=0.72$. This object is marginally resolved in the deep NOT image. Taking into account the problems with the VLT data (e.g. variable PSF, saturated core) the results agree very well with each other. In comparison to W96, this data gives about a 0.6 mag brighter host galaxy. Also the environment of this object is well studied (Benitez et al. 1996; Yanny et al. 1997).

$\mathbf{1 1 4 7 + 2 4 5}$ In previous studies (S93 and SU00) this object remained unresolved as well as in the present NOT image. The PSF was constructed using the core of a faint star $\left(m_{R} \sim 18\right)$ and the wings from other frame during the same observing run. The redshift of this object is unknown, hence the present data suggest high redshift.

1308+326 The emission line strengths are reported to be variable in this object (Scarpa \& Falomo 1997; S93 and references therein). Scarpa \& Falomo questioned the classification of this object as a BL Lac. In a previous imaging study, S93 stated that the object is not completely stellar. The best fit de Vaucouleurs model gives $m_{\text {host }}=20.84$, $r_{\mathrm{e}}=0.68,\left(M_{\text {host }}=-26.16, r_{\mathrm{e}}=7.4 \mathrm{kpc}\right)$ with $\chi^{2}=0.53$, however the data does not fit into the Fundamental Plane (2.5 mag above the best fit), hence this object is judged to be unresolved.

$\mathbf{1 4 1 8}+\mathbf{5 4 6}$ The morphology of the host galaxy of this object (OQ530) has been under debate since A91 claimed it to have a disc-like host. Later, S93 preferred elliptical, W96 again disc-type and most recently SU00 an elliptical host. The present NOT data prefers an elliptical host. As discussed in Appendix B, the galaxy parameters depend on the length of the extracted profile. In this case, $r_{\mathrm{e}}$ decreased as the image gets deeper, which is inconsistent with the smaller $r_{\mathrm{e}}$ compared to the HST and NOT data. Most likely the confusion in the host galaxy's morphology is due to an extra component in the nucleus of this object, as was proposed by S93.

1514-241 This object (AP Lib) is one of the closest ( $z=0.049)$ objects in the 1 Jy sample and the surrounding nebulosity was detected some time ago (e.g. Disney et al. 1974). The host has been found to be a round elliptical galaxy (A91, S93, SU00) and the present NOT data confirms the parameters found before. In $H$-band observations Kotilainen et al. (1998) found the host to be elliptical with $M_{H}=-25.1$ and $r_{\mathrm{e}}=3.3 \mathrm{kpc}$. $\mathrm{S} 93$ suggested tidal interaction with a companion galaxy, which could be the reason for the extra light seen in the tail of the profile (Fig. 1).

1538+149 This distant object $(z=0.605)$ was reported to be slightly resolved in the S93 $B$-band image and marginally resolved by W96. The host galaxy remained unresolved in NIR by Kotilainen et al. (1998). In the HST pointed observations, this object was resolved (U99) and barely resolved by SU00. In the NOT data this object is resolved, but both an elliptical and disc-type host give an equally good fit (Table 2). Due to technical reasons the $R$-band CCD frame was binned $2 \times 2$, hence the pixel size was $0 . \prime 352$.

$\mathbf{1 6 5 2}+398$ This nearby object $(z=0.033)$ is also well studied (Mkn 501). The proposed size of the host galaxy has varied from 9 to 20 arcsec (Table 4). The present data prefers the larger value. However, using the same data but a two-dimensional analysis, N99 found a somewhat smaller host galaxy. It should be noted that there are large residuals near the core, which could indicate an extra component. This would also give an explanation why the size of the galaxy varies so drastically in different studies (i.e. the different depth of the image, see Fig. B.1).

$\mathbf{1 7 4 9 + 0 9 6}$ This object was resolved by S93, W96 and SU00. Due to the low galactic latitude the field is crowded with stars, which might contaminate the tail of the profile. S93 could not define the brightness of the host galaxy and there is a discrepancy between the W96 and SU00 results. The morphology remains uncertain in this object, but the NOT data favours SU00, a fainter and smaller host galaxy than W96's.

1749+701 This object $(z=0.770)$ has been studied by S93 and SU00, but remained unresolved. Also in the present NOT data the profile is indistinguishable from the PSF.

1803+784 This object $(z=0.680)$ has been studied by S93 and SU00, but remained unresolved. Also in the present NOT data the profile is indistinguishable from the PSF.

$\mathbf{1 8 0 7 + 6 9 8}$ One of the nearby $(z=0.051)$ well studied BL Lacs (3C371) with an elliptical host galaxy (e.g. Miller 1975). This is an interacting system (S93) and one of the rare cases which has an optical jet (Nilsson et al. 1997). The present results agree with the previous ground based imaging, but SU00 found considerably 
smaller $r_{\mathrm{e}}$. In this object it was found that $r_{\mathrm{e}}$ depends strongly on the length of the extracted profile. This reduces the apparent discrepancy between the HST and NOT data. It suggests that this object might also have an extra component in the nucleus or it could be due to the tidal interaction with the neighbouring galaxy.

$\mathbf{1 8 2 3}+\mathbf{5 6 8}$ This is the most distant $(z=0.664)$ resolved object. The profile is consistent either with de Vaucouleurs or disc models (Table 2). Taking into account the errors, the NOT and HST data (SU00) agree quite well.

$\mathbf{2 0 0 7 + 7 7 7}$ This object was found unresolved by S93, marginally resolved by W96 and resolved by SU00. Yet the HST data was not deep enough to discriminate between an elliptical and disc model. Neither de Vaucouleurs nor exponential disc fits are perfect using the present NOT data. In the former case the tail $\left(r>2^{\prime \prime}\right)$ is too bright and on the other hand the disc is too faint after $r>2^{\prime \prime}$ (Fig. 1). Deeper images are needed in order to discriminate between the two models.

2131-021 This distant ( $z=1.285)$ object has remained unresolved in previous studies (S93, SU00) and also in the present work. The best fit de Vaucouleurs model gives slightly better $\chi^{2}$ value $m_{\text {host }}=21.9 ; r_{\mathrm{e}}=22^{\prime \prime} 18,\left(M_{\text {host }}=\right.$ $-26.75 ; r_{\mathrm{e}}=26 \mathrm{kpc}$ ) than pure point source, however the data does not fit (2.5 mag above the best fit) to the FP (Fig. 7), hence this object is classified as unresolved.

$\mathbf{2 2 0 0}+\mathbf{4 2 0}$ (BL Lacertae) This is the prototype of BL Lac objects and it is hosted with an elliptical galaxy (S93, W96, SU00). The field is crowded with galactic stars due to the low galactic latitude, and a nearby bright star hampers extracting the profile at long distances $\left(r>10^{\prime \prime}\right)$. The fitting was done using the eastern part of the profile (from the major axis) up to $21^{\prime \prime}$. Agreement with the present results and S93 improves when adjusting the length of the profile. SU00 found a slightly fainter host galaxy than in the present work.

2240-260 The previous (S93, SU00) imaging studies have failed to resolve this $(z=0.774)$ object as well as the present NOT data.

2254+074 This object was the most distant well resolved one by S93 $(z=0.194)$. All the previous imaging studies (S93, W96, Falomo 1996, SU00) agree well with each other and in all cases elliptical host have been preferred. Kotilainen et al. (1998) resolved the host galaxy in NIR $\left(M_{H}=-25.6, r_{\mathrm{e}}=32.5 \mathrm{kpc}\right)$. Unfortunately dust hampered the NOT observations.

\section{Appendix B: Galaxy parameters vs. fitting radius}

Usually the differences of the derived host galaxy properties are much larger than the expected scatter from the simulations. One possible explanation for this is the deepness of the image, especially if the profile does not exactly follow de Vaucouleurs (or disc) profile or there might be an extra component in the core of the galaxy. The presented deep images of the low redshift objects enable us to study this issue in more detail than before.

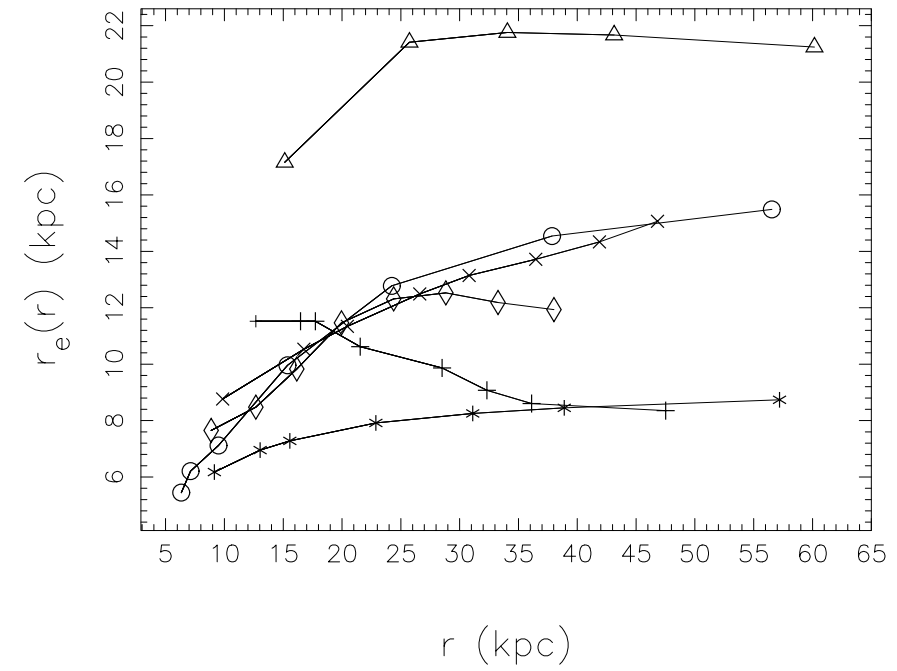

Fig. B.1. The dependence of the derived $r_{\mathrm{e}}$ as a function of the length of the galaxy profile. The objects and symbols are: $2254+074(\triangle)$, Mkn $501(\circ)$, AP Lib (*), 3C371 (×), OQ $530(+)$, BL Lac $(\diamond)$.

For the low-redshift $(z \lesssim 0.2)$ objects, the present NOT data is suitable for studying the length of the extracted radius versus the derived galaxy properties. Figure B.1 shows how the derived half light radii $\left(r_{\mathrm{e}}\right)$ change, as there is a stronger contribution from the tail of the galaxy profile. In all but one case (OQ530) the values of $r_{\mathrm{e}}$ increase as the profile becomes longer (i.e. goes deeper). Usually beyond a radius of $\sim 30 \mathrm{kpc}$ the $r_{\mathrm{e}}$ stabilizes and the changes are less than $10 \%$. A similar pattern is seen (a steady increase) when comparing brightness with the increasing in length of the profile. However, the $m_{\text {host }}$ varies with an amplitude of $\sim 0.3$ mag. The agreement between the W96 and the NOT data is improved using a $\sim 8^{\prime \prime}$ profile for the analysis. Also, better consistency is achieved between the NOT data and S93, when the fitting was started at the point where the scaled PSF's surface brightness was about two magnitudes fainter than the galaxy's.

\section{References}

Abraham, R. G., McHardy, I. M., \& Crawford, C. S. 1991, MNRAS, 252, 482 (A91)

Beckmann, V., Bade, N., \& Wucknitz, O. 1999, A\&A, 352, 395

Benitez, E., Dultzin-Hacyan, D., Heidt, J., et al. 1996, ApJ, 464, L47

Blandford, R. D., \& Rees, M. J. 1978, in Pittsburgh Conference on BL Lac Objects, ed. A. N. Wolfe (University of Pittsburgh Press), 328

Bressan, A., Chiosi, C., \& Fogotto, F. 1994, ApJS, 94, 63

Browne, I. W. A., \& Marchã, M. J. M. 1993, MNRAS, 261, 795

Caon, N., Capaccioli, M., \& D'Onofrio, M. 1993, MNRAS, 265, 1013

Coleman, G. D., Wu, C. C., \& Weedman, D. W. 1980, ApJS, 43,393

de Juan, L., Colina, L., \& Golombek, D. 1996, A\&A, 305, 776 
Disney, M. J., Peterson, B. A., \& Rodgers, A. W. 1974, ApJ, 194, L79

Djorgovski, S., \& Davis, M. 1987, ApJ, 313, 59

Drinkwater, M. J., Webster, R. L., Francis, P. J., et al. 1997, MNRAS, 284, 85

Efstathiou, G., Ellis, R. S., \& Peterson, B. A. 1988, MNRAS, 232,431

Falomo, R. 1996, MNRAS, 283, 241

Falomo, R., Pesce, J. E., \& Treves, A. 1995, ApJ, 438, L9

Falomo, R., Urry, C. M., Pesce, J. E., et al. 1997a, ApJ, 476, 113

Falomo, R., Kotilainen, J., Pursimo, T., et al. 1997b, A\&A, 321,374

Falomo, R., \& Kotilainen, J. 1999, A\&A, 352, 85 (FK99)

Feigelson, E. D., \& Nelson, P. I. 1985, ApJ, 293, 192

Fiorucci, M., \& Tosti, G. 1996, A\&AS, 116, 403

Fiorucci, M., Tosti, G., \& Rizzi, N. 1998 PASP, 110105

Fossati, G., Maraschi, L., Celotti, A., Comastri, A., \& Ghisellini, G. 1998, MNRAS, 299, 433

Fukugita, M., Shimasaku, K., \& Ichikawa T. 1995, PASP, 107, 945

Govoni, F., Falomo, R., Fasano, G., \& Scarpa., R. 2000, A\&A, 353,507

Heidt, J. 1999, in ASP Conf. Proc. BL Lac Phenomonen, ed. L. O. Takalo, \& A. Sillanpää (San Francisco: ASP), 367

Heidt, J., Nilsson, K., Pursimo, T., Takalo, L. O., \& Sillanpää, A. 1996, A\&A, 312, L13

Heidt, J., Nilsson, K., Sillanpää, A., Takalo, L. O., \& Pursimo, T. 1999a, A\&A, 341, 683

Heidt, J., Nilsson, K., Appenzeller, I., et al. 1999b, A\&A, 352, L11

Hutchings, J. B., Crampton, D., Campbell, B., Duncan, D., \& Glendenning, B. 1984, ApJS, 55, 319

Isobe, T., Feigelson, E. D., \& Nelson, P. I. 1986, ApJ, 306, 490

Jaffe, W., Holland, C. F., Ferrares, L., van den Bosch, F., \& O'Connell, R. W. 1993, Nature, 364, 213

Kormendy, J. 1977, ApJ, 218, 333

Kotilainen, J. K., Falomo, R., \& Scarpa, R. 1998, A\&A, 336, 479

Kühr, H., Witzel, A., Pauliny-Toth, I. I. K., \& Nauber, U. 1981, A\&AS, 45, 367

Landolt, A. U. 1983, AJ, 88, 853

Landolt, A. U. 1993, AJ, 104, 340

Laurent-Muehleisen, S. A., Kollgaard, R. I., Feigelson, E. D., Brinkmann, W., \& Siebert, J. 1999, ApJ, 525, 127

Lawrence, C. R., Zucker, J. R., Readhead, A. C. S., et al. 1996, ApJS, 107, 541

McHardy, I. M., Abraham, R. G., Crawford, C. S., et al. 1991, MNRAS, 249, 742
McLure, R. J., Kukula, M. J., Dunlop, J. S., et al. 1999, MNRAS, 308, 377

Miller, J. 1975, ApJ, 200, L55

Morris, S. L., Stocke, J. T., Gioia, I. M., et al. 1991, ApJ, 380, 49

Nilsson, K., Charles, P. A., Pursimo, T., et al. 1996, A\&A, 314, 754

Nilsson, K., Heidt, J., Pursimo, T., et al. 1997, ApJ, 484, L107

Nilsson, K., Pursimo, T., Takalo, L. O., et al. 1999, PASP, 111, $1223(\mathrm{~N} 99)$

Ostriker, J. P., \& Vietri, M. 1985, Nature, 318, 446

Padovani, P. 1999, in ASP Conf. Proc. BL Lac Phenomonen, ed. L. O. Takalo, \& A. Sillanpää (San Francisco: ASP), 339

Padovani, P., \& Giommi, P. 1995, ApJ, 444, 567

Pursimo, T., Nilsson, K., Sillanpää, A., Takalo, L. O., \& Heidt, J. 1999 in ASP Conf. Proc. BL Lac Phenomonen, ed. L. O. Takalo, \& A. Sillanpää (San Francisco: ASP), 385

Pursimo, T., et al. 2001, A\&A, submitted

Scarpa, R., \& Falomo, R. 1997, A\&A, 325, 109

Scarpa, R., Urry, C. M., Falomo, R., Pesce, J. E., \& Treves, A. 2000, ApJ, 532, 740 (SU00)

Schade, D., Boyle, B. J., \& Letawsky, M. 2000, MNRAS, 315, 498

Schull, J. M., \& Van Steenberg, M. E. 1985, ApJ, 294, 599

Smith, P. S., Balonek, T. J., Heckert, P. A., Elston, R., \& Schmidt, G. D. 1985, AJ, 90, 1184

Stark, A. A., Gammie, C. F., Wilson, R. W., et al. 1992, ApJS, 79,77

Stickel, M., Padovani, P., Urry, C. M., Fried, J. W., \& Kühr H. 1991, ApJ, 374, 431

Stickel, M., Fried, J. W., \& Kühr H. 1993, A\&AS, 98, 393 (S93)

Stickel, M., Meisenheimer, K., \& Kühr, H. 1994, A\&AS, 105, 211

Stocke, J. T., Wurtz, R., \& Perlman, E. S. 1995, ApJ, 454, 55

Stocke, J. T., \& Rector, T. A. 1997, ApJ, 489, L17

Urry, C. M., \& Padovani, P. 1995, PASP, 107, 803

Urry, C. M., Falomo, R., Scarpa, R., et al. 1999, ApJ, 512, 88 (U99)

Urry, C. M., Scarpa, R., O'dowd, M., et al. 2000, ApJ, 532, 816 (U00)

Villata, M., Raiteri, C. M., Lanteri, L., Sobrito, G., \& Cavallone, M. 1998, A\&AS, 130, 305

Wright, S. C., McHardy, I. M., \& Abraham, R. G. 1998, MNRAS, 295, 799

Wurtz, R., Stocke, J. T., \& Yee, H. K. C. 1996, ApJS, 103, 109 (W96)

Yanny, B., Jannuzi, B. T., \& Impey, C. 1997 ApJ, 484, L113 